九州大学学術情報リポジトリ

Kyushu University Institutional Repository

\title{
YAP signaling induces PIEZ01 expression to promote oral squamous cell carcinoma cell proliferation
}

\section{Hasegawa, Kana}

Laboratory of Oral Pathology, Division of Maxillofacial Diagnostic and Surgical Sciences, Faculty of Dental Science, Kyushu University

Fujii, Shinsuke

Laboratory of Oral Pathology, Division of Maxillofacial Diagnostic and Surgical Sciences, Faculty of Dental Science, Kyushu University

Matsumoto, Shinji

Department of Molecular Biology and Biochemistry, Graduate School of Medicine, Osaka University

\section{田尻，祐大}

Laboratory of Oral Pathology, Division of Maxillofacial Diagnostic and Surgical Sciences, Faculty of Dental Science, Kyushu University

他

ht tp://hdl. hand le. net/2324/4481560

出版情報：Journal of Pathology. 253 (1)，pp.80-93，2021-01. Wiley バージョン：

権利関係: 


\title{
YAP signaling induces PIEZO1 expression to promote oral squamous cell carcinoma cell proliferation
}

\begin{abstract}
A short running title: YAP signaling induces PIEZO1 expression to promote OSCC cell growth
\end{abstract}

\author{
Kana Hasegawa $^{1}$, Shinsuke Fujii ${ }^{1 *}$, Shinji Matsumoto ${ }^{2}$, Yudai Tajiri ${ }^{1,3}$, Akira Kikuchi ${ }^{2}$ \\ and Tamotsu Kiyoshima ${ }^{1}$
}

${ }^{1}$ Laboratory of Oral Pathology, Division of Maxillofacial Diagnostic and Surgical Sciences, Faculty of Dental Science, Kyushu University, 3-1-1 Maidashi, Higashi-ku, Fukuoka 812-8582, Japan

${ }^{2}$ Department of Molecular Biology and Biochemistry, Graduate School of Medicine, Osaka University, 2-2 Yamadaoka, Suita 565-0871, Japan

${ }^{3}$ Department of Dentistry and Oral Surgery, Clinical Research Institute, National Hospital Organization Kyushu Medical Center, 1-8-1 Jigyohama, Chuo-ku, Fukuoka 810-8563, Japan

*Corresponding author. Laboratory of Oral Pathology, Division of Maxillofacial Diagnostic and Surgical Sciences, Faculty of Dental Science, Kyushu University

3-1-1 Maidashi, Higashi-ku, Fukuoka 812-8582, Japan

Phone: +81-92-642-6328; Fax: +81-92-642-6329

E-mail: sfujii@dent.kyushu-u.ac.jp 
Conflict of interest statements: No conflicts of interests were declared.

A word count: 3,998

DNA microarray analysis was carried out in the current study, and the raw data reported in this study was deposited in NCBI GEO under accession number (GSE150044). 


\begin{abstract}
Most cancer cells are exposed to extracellular environments, such as an increase in extracellular matrix (ECM) stiffness with genetic transformation of cancer cells and soluble signals consisting of growth factors and cytokines. It is therefore conceivable that changes in the tumorous extracellular environments would affect tumor cell behavior. The Hippo pathway reportedly responds to the extracellular environment and regulates the nuclear localization of the transcription co-activator, yes-associated protein (YAP)/transcriptional co-activator with PDZ-binding motif (TAZ). Inactivation of the Hippo pathway with nuclear translocation of YAP/TAZ leads to stimulate cell proliferation. Its pathway also regulates gene expression, but the precise molecule meditating the cell-proliferating effect of YAP signaling on oral squamous cell carcinoma (OSCC) is unclear. First, we examined the effects of YAP signaling on OSCC tumorigenesis. Loss-of-function experiments using siRNA or an inhibitor, and immunohistochemical analyses of tissue specimens obtained from OSCC patients demonstrated that YAP signaling was involved in OSCC cell proliferation. Second, we identified Piezo-type mechanosensitive ion channel component 1 (PIEZO1), a $\mathrm{Ca}^{2+}$ channel, as a transcriptional target of YAP signaling and showed that an elevated PIEZO1 expression was required for PIEZO1 agonist-dependent $\mathrm{Ca}^{2+}$ entry and cell proliferation in OSCC cells. Furthermore, experiments using three-dimensional culture and suspension culture revealed that PIEZO1, the expression of which is regulated by YAP signaling, was involved in OSCC cellular growth. Finally, YAP overexpression in the nucleus and/or cytoplasm was immunohistochemically detected in tumor lesions with high
\end{abstract}


frequent expression of both PIEZO1 and Ki-67 but not in non-tumor regions of OSCC specimens. These results suggest that the YAP/PIEZO1 axis promotes OSCC cell growth.

Key words: OSCC, YAP, PIEZO1, Proliferation, Three-dimensional culture, Suspension culture 


\section{Introduction}

Oral squamous cell carcinoma (OSCC) is the $11^{\text {th }}$-most common cancer in the world [1], with over 300,000 new cases and 145,000 deaths every year [2]. The 5-year survival rate of OSCC patients is approximately 50\% [3]. Although treatments for OSCC, including surgical resection, radiotherapy and chemotherapy, have been improving, the rate of recurrence is $20 \%-40 \%$, resulting in a reduced survival rate $[4,5]$. Therefore, a novel cancer therapy based on the molecular mechanisms underlying OSCC tumorigenesis is awaited.

Tumor cells are exposed to the extracellular environments, such as ECM and soluble signals consisting of growth factors and cytokines [6]. Clinically, matrix stiffening is a prominent hallmark of the tumor microenvironment. In OSCC, stiffness is an important clinical feature. Recent studies showed novel mechanotrasduction mechanisms, in which an increase in tumorous ECM stiffness drove tumor progression through the activation of intracellular signaling, such as $\beta 1$ integrin-FAK signaling and RHOA-AKT-p300 signaling in breast cancer and liver cancer, respectively [7,8]. We also demonstrated that transient receptor potential vanilloid 4 (TRPV4), a $\mathrm{Ca}^{2+}$ channel, responds to the extracellular stiffness, which in turn promoting OSCC cell proliferation through CaMKIIAKT activation [9]. These reports indicate that tumorous extracellular environments could affect tumor cell behavior through several mechanotrasduction machineries.

The Hippo pathway responds to the extracellular environments and stimulates cell proliferation, through regulating the nuclear localization of major downstream effectors, yes-associated protein (YAP)/transcriptional co-activator with PDZ-binding motif(TAZ), 
resulting in the induction of transcriptional target expression [10-13]. Its pathway has been involved in tumorigenesis in OSCC and hepatocellular carcinoma and is associated with the poor prognosis in breast cancer, ovarian cancer and hepatocellular carcinoma [14-17], but transcriptional targets that mediate the function of the Hippo pathway are not well understood.

Piezo-type mechanosensitive ion channel component (PIEZO) channels, including PIEZO1 and PIEZO2, were identified as mechanically activated ion channels [18]. PIEZO1 is reportedly associated with epithelial cellular division, neural cell differentiation and vasculature development by sensing mechanical stress, such as cell density, substrate stiffness and shear stress [19-21]. Although the effects of PIEZO1 on tumorigenesis have been demonstrated [22-25], its function and expression mechanism in OSCC tumorigenesis remain unclear. Recent reports showed that the depletion of PIEZO1 expression induced nuclear exclusion of YAP in human neural stem cells and zebrafish outflow tract valve development $[20,26]$, suggesting that PIEZO1 could act as an upstream of YAP. But, the hierarchical relationship between YAP and PIEZO1 is unclear in OSCC. We conducted a study to clarify their relationship in OSCC and elucidate the function of PIEZO1 to control OSCC cellular growth using cell lines and pathological specimens. 


\section{Materials and methods}

\section{Cell lines and reagents}

Human OSCC cell lines HSC-4, HSC-3, HSC-2, SAS (Japanese Cancer Research Resources Bank), MISK81-5 and sMISK [27], human gastric cancer cell line MKN-45 (Japanese Cancer Research Resources Bank) and human lung adenocarcinoma cell line A549 [28] were used in this study. HSC-4, HSC-3, HSC-2, MISK81-5 and sMISK were maintained in $\alpha$-MEM (Invitrogen, Carlsbad, CA, USA), SAS and A549 were maintained in D-MEM (Invitrogen), and MKN-45 was maintained in RPMI-1640 (Invitrogen). These culture media were supplemented with 10\% FBS (Invitrogen), and contained $100 \mathrm{IU} / \mathrm{ml}$ penicillin and $100 \mathrm{mg} / \mathrm{ml}$ streptomycin (Invitrogen). All these cell lines were incubated at $37^{\circ} \mathrm{C}$ in a $5 \% \mathrm{CO}_{2}$ atmosphere. See supplementary Materials and methods for further details.

\section{Knockdown of protein expression by siRNA and quantitative RT-PCR}

The effects of protein knockdown by siRNA were analyzed as previously described [29]. Briefly, siRNAs (final conc. $20 \mathrm{nM}$ ) were transfected into OSCC cells using Lipofectamine RNAiMAX (Invitrogen). Target sequences are listed in supplementary material (Table S1). The transfected cells were then used for experiments conducted at $48 \mathrm{~h}$ post-transfection.

Quantitative RT-PCR was performed as described previously [29]. Primers are listed in supplementary material (Table S2). 


\section{Plasmid construction and infection using lentivirus harboring a cDNA or shRNA}

The YAP ${ }^{5 S A}$ plasmid, CSII-CMV-MCS-IRES2-Bsd/FLAG-YAP5SA, was used [12]. To construct a lentiviral vector harboring shRNA, a DNA fragment containing the H1 promoter and shRNA was cloned into CS-RfA-EVBsd, which was kindly provided by Dr. H. Miyoshi (RIKEN BioResource Center, Ibaraki, Japan) [30], using Gateway technology (Invitrogen). Target sequence is \#1 5'-AGAAGAAGATCGTCAAGTA-3' or \#2 5'- ACCAACCTCATCAGCGACT-3'. The vectors were then transfected along with the packaging vectors, pCAG-HIV-gp and pCMV-VSV-G-RSV-Rev, into X293T cells using the Lipofectamine LTX reagent (Invitrogen) to generate lentiviruses [28]. See supplementary Materials and methods for further details.

\section{Three-dimensional culture and suspension culture}

3D culture was prepared using collagen gel culture kit Tri-D (Nippi, Inc.) or Matrigel (BD Biosciences, San Jose, CA, USA) according to the manufacturer's protocol. Briefly, bottom collagen gel $(1 \mathrm{mg} / \mathrm{ml})$ was poured into the dishes and incubated for polymerization for $30 \mathrm{~min}$ at $37^{\circ} \mathrm{C}$. Then, cells mixed with $1 \mathrm{mg} / \mathrm{ml}$ collagen gel were layered onto the bottom gel. Culture medium was added on top, and then cells cultured in $3 \mathrm{D}$ culture were incubated at $37^{\circ} \mathrm{C} .40 \mu \mathrm{l}$ of Matrigel was mounted on a round coverslip and incubated for $30 \mathrm{~min}$ at $37^{\circ} \mathrm{C}$ to solidify the gel, and cells $\left(4 \times 10^{4}\right.$ cells $)$ suspended in $1 \mathrm{ml}$ of growth medium containing 2\% Matrigel (v/v) were incubated for $96 \mathrm{~h}[9,31]$. 
In suspension culture, the cells $\left(2 \times 10^{5}\right.$ cells $)$ were cultured with growth medium in low-attachment $1.5 \mathrm{ml}$ tube (Sumitomo Bakelite Co., Ltd, Tokyo, Japan) for $24 \mathrm{~h}$.

\section{Cell proliferation assay}

To examine proliferative capabilities in 2D culture, 3D culture or suspension culture, CyQUANT NF assay (Invitrogen) was performed according to the manufacturer's protocol. Then, the fluorescence intensity was measured using FlexStation 3 microplate reader (Molecular Devices, Tokyo, Japan) at $485 \mathrm{~nm}$ excitation/530 nm emission.

\section{ChIP assay}

ChIP assay was performed as described previously [32]. HSC-4 cells $\left(1 \times 10^{7}\right)$ were crosslinked with $1 \%(\mathrm{v} / \mathrm{v})$ formaldehyde for $20 \mathrm{~min}$ at room temperature. The cells were lysed and sonicated to shear DNA to a size range between 200 and $1000 \mathrm{bp}$. Sheared chromatin samples were incubated for $4 \mathrm{~h}$ at $4^{\circ} \mathrm{C}$ with $10 \mu \mathrm{g}$ of antibodies. Immunocomplexes were absorbed with salmon sperm DNA/protein A-agarose beads. See supplementary Materials and methods for further details.

\section{Intracellular calcium measurement}

Intracellular calcium measurement was performed as described previously [9]. See supplementary Materials and methods for further details.

\section{Immunocytochemistry}


Immunocytochemistry was carried out as previously described [33]. See supplementary Materials and methods for further details.

\section{Patients and immunohistochemistry}

A total of 45 patients with ages ranging from 42 to 90 years (median, 70 years) with primary OSCC diagnosed for treatment at the Department of Oral and Maxillofacial Surgery, Kyushu University Hospital, Japan, from March 2011 to February 2020 were examined in this study. The protocol for this study was approved by the ethical review board of the Local Ethical Committee of Kyushu University, Japan (\#30-235). Following the initial biopsy, all specimens were fixed in $10 \%(\mathrm{v} / \mathrm{v})$ formalin and embedded in paraffin blocks. Subsequently, the paraffin-embedded specimens were sliced into $4-\mu \mathrm{m}-$ thick sections, stained with hematoxylin-eosin (HE), and examined by three experienced pathologists to confirm the diagnoses and the histological grades. The histological grade in the OSCC was assessed according to the WHO classification [34]. The tumor extent and the clinical stages were evaluated according to the TNM classification established by the American Joint Committee on Cancer and the International Union Against Cancer (UICC) [35]. The medical records were reviewed to collect information on the clinical characteristics. The clinicopathological data of the patients with OSCC are presented in Table 1 and 2. Immunohistochemical staining was performed as previously described [9]. See supplementary Materials and methods for further details.

\section{Microarray analysis}


Microarray analyses were performed using HSC-4 cells cultured in 2D culture or suspension culture as previously described [33]. The raw data reported in this study was deposited in NCBI GEO under accession number (GSE150044). Gene Ontology (GO) analysis was performed using DAVID database (http://david.abcc.ncifcrf.gov/). See supplementary Materials and methods for further details.

\author{
Analysis of TCGA head and neck cancer dataset \\ PIEZO1, PIEZO2, amphiregulin (AREG) and cyclin dependent kinase 6 (CDKO) gene \\ expression data for 518 head and neck tumor lesions and 44 non-tumor regions were \\ obtained from The Cancer Genome Atlas (TCGA) web site (http://tcga- \\ data.nci.nih.gov/tcga/tcgaHome2.jsp).
}

\title{
Statistical analysis
}

Significant differences were determined using Fisher's exact test for Table 1 and 2. For other experiments, significant differences were determined using Student's t test and oneway ANOVA with turkey. $P$ value of $<0.05$ or 0.01 was considered statistically significant. 


\section{Results}

\section{Proliferative capabilities of oral squamous cell carcinoma cells in three-dimensional and suspension culture}

Inactivation of the Hippo pathway regulates YAP/TAZ nuclear translocation, thereby inducing transcriptional targets and promoting cell proliferation [10-17]. YAP/TAZ siRNA and the treatment with simvastatin, a YAP inhibitor [36,37], reduced not only the mRNA levels of ankyrin repeat domain1 (ANKRD1) and cellular communication network factor 1 (CCN1; also known as CYR61), which are transcriptional targets of YAP/TAZ, but also 2-dimentional (2D) proliferation of HSC-4 cells (supplementary material, Figure S1A). These loss-of-function experiments indicated that YAP/TAZ signaling was involved in HSC-4 cell proliferation. Next, we conducted to set up the cell culture system, which is capable of analyzing the relationship between YAP/TAZ signaling and its transcriptional targets.

Our recent study showed that HSC-4 cells respond to extracellular environments, such as 3D culture with Matrigel and 2D plastic dish culture, and exhibited lower proliferation capabilities in $3 \mathrm{D}$ culture than in $2 \mathrm{D}$ culture [9]. Consistently, the cellular growth in $3 \mathrm{D}$ culture with collagen gel was also suppressed compared with 2D culture in HSC-4 and HSC-3 cells (Figure 1A), indicating that extracellular conditions do indeed affect OSCC cellular proliferation capabilities, regardless of the matrix. Proliferative capabilities in suspension culture were extremely lower than 2D or 3D culture (Figure 1A). As YAP signaling could be inactivated in suspension culture [12], we carried out a microarray analysis using HSC-4 cells cultured in 2D and suspension culture. Expectedly, GO 
analysis showed enrichment for previously reported the YAP/TAZ function, such as DNA replication, mitotic nuclear division, DNA replication initiation, regulation of cell cycle, cell proliferation, DNA repair, cell cycle and positive regulation of cell migration (Figure 1B and supplementary material, Table S3). Indeed, transcriptional targets of YAP/TAZ, such as AREG, aurora kinase B (AURKB), cyclin D1(CCND1), CDK6, E2F transcription factor 1 (E2F1), forkhead box M1(FOXM1), kinesin family member 23 (KIF23) and minichromosome maintenance complex component 7 (MCM7) [38-44] were listed as a down-regulated gene in suspension culture (supplementary material, Table S4). These data suggested that suspension culture could be useful to identify transcriptional targets of YAP/TAZ signaling. In suspension culture, YAP/TAZ expression was reduced in a time-dependent manner (Figure1C). Similarly, the mRNA levels of ANKRD and CYR61 were reduced in OSCC cells in suspension culture compared with 2D culture (Figure 1D and supplementary material, Figure S1B). In addition, siRNA treatment for large tumor suppressor 2 (LATS2), which phosphorylates YAP/TAZ on serine residues [10], reversed the suspension culture-dependent decrease of ANKRD1, CYR61, AURKB and FOXM1 expression (Figure 1E and supplementary material, Figure S1C and S1D). These results indicate that YAP/TAZ signaling may be inactivated in the suspension culture, thereby suppressing cell proliferation in OSCC cells.

\section{PIEZO1 is a transcriptional target of YAP signaling in oral squamous cell carcinoma cells}

PIEZO channels, including PIEZO1 and PIEZO2, were identified as mechanically 
activated ion channels [18]. The Cancer Genome Atlas (TCGA) dataset showed that PIEZO1 and PIEZO2 mRNA were elevated in tumor lesions compared with in non-tumor regions, but PIEZO2 mRNA expression levels were lower than that of PIEZO1 in head and neck region (supplementary material, Figure S2A). Although PIEZO1 has been demonstrated both positive [22,23] and negative [24,25] effects on tumorigenesis, its function and expression mechanism in OSCC tumorigenesis remain unclear. Therefore, the function of PIEZO1 in OSCC tumorigenesis was investigated in the current study. Previous report showed YAP activation in OSCC tumor lesion [37]. Consistently, the expression of $A R E G$ or CDK6 was elevated in tumor lesions. Since a significant correlation between PIEZOI and AREG or CDK6 was observed (supplementary material, Figure S2A), it is possible that PIEZO1 expression is correlated with YAP signaling. It has been reported that PIEZO1 could act as an upstream of YAP in the developmental stages $[20,26]$. Therefore, we conducted to clarify the relationship between YAP/TAZ signaling and PIEZO1 in OSCC cells in the subsequent experiments.

PIEZO1 expression was decreased in 3D culture compared with 2D culture in HSC-4 cells (Figure 2A), and was also reduced in suspension culture (Figure 2B and supplementary material, Figure S2B). Importantly, knockdown of LATS2 rescued the suspension culture-dependent reduction of the expression of PIEZO1 in HSC-4 cells (Figure 2C), suggesting that YAP/TAZ signaling may act as an upstream of PIEZO1. In addition, two different YAP/TAZ siRNAs reduced PIEZO1 expression in HSC-4 and HSC-3 cells (Figure 2D and supplementary material, Figure S2C). To elucidate the function of YAP, HSC-4 and HSC-3 cells expressing mock or an active YAP mutant 
YAP5SA, in which five possible phosphorylation serine residues are changed to alanine $[12,45]$, were generated by lentiviral transduction. In YAP ${ }^{5 \mathrm{SA}}$-expressing OSCC cells, the expression of PIEZO1, as well as ANKRD1 and CYR61, was elevated compared with mock-expressing cells, indicating that YAP activation induces PIEZO1 expression (Figure 2E and supplementary material, Figure S2C and S2D). We conducted to find the putative binding sites of TEA domain transcription factors 4 (TEAD4), which is a major transcription factor in the Hippo pathway regulating the expression of transcriptional targets associated with YAP/TAZ [46], using Cistrome Data Browser database (http://cistrome.org/db/\#/) in human PIEZO1 gene. The database showed that ChIP-seq peaks of TEAD4 and active histone modifications, such as H3K27ac and H3K9ac, were hardly detected in the region upstream from the TSS of PIEZO1 gene. In contrast, three ChIP-seq peaks of TEAD1 or TEAD4, which are commonly detected in multiple cell lines and contain putative binding sites of four TEAD genes (data not shown), were found within the PIEZO1 gene body. Site 1 is located within exon 1, and site 2 and site 3 were located between exons 1 and 2. In addition, these sites overlapped the peaks of H3K27ac and $\mathrm{H} 3 \mathrm{~K} 9 \mathrm{ac}$, and exhibited high enhancer activity region using epilogos database (https://epilogos.altius.org) (supplementary material, Figure S2E). ChIP assay revealed that TEAD4 indeed bound to PIEZO1 gene more dominantly through sites 2 and 3, as well as to promoter region of $A N K R D 1$ as a positive control [47], than in site 1 (Figure 2F and supplementary material, Figure S2E). Furthermore, YAP5SA also formed a complex with sites 2 and 3 (Figure 2F). Previous report showed that YAP activity is mediated via TEADs binding to not only promoters but also enhancers of the 
transcriptional targets [47]. Therefore, the current TEAD4 binding sites could be involved in PIEZO1 expression mechanism. These results suggest that PIEZO1 is a possible transcriptional target of YAP signaling.

\section{PIEZO1 expression is involved in agonist-induced ERK1/2 and p38 MAPK activation}

PIEZO1 or PIEZO2 expression in cancer cell lines was analyzed using Cancer Cell Line Encyclopedia (https://portals.broadinstitute.org/ccle) dataset, which was viewed by cBioportal (https://www.cbioportal.org). In HSC-2, HSC-3 and HSC-4 cells, PIEZO1 was expressed to the similar levels to other cancer cells, while PIEZO2 expression was little expressed in many cancer cells (supplementary material, Figure S3A). We also measured PIEZO1 expression in six human OSCC cell lines and a control gastric cancer cell line. HSC-4 and HSC-3 highly expressed PIEZO1 (Figure 3A), while PIEZO2 mRNA level was low in OSCC cell lines, compared with A549 cells (supplementary material, Figure S3B). Treatment with Yoda1, a specific PIEZO1 agonist $[48,49]$, increased the intracellular $\mathrm{Ca}^{2+}$ influx in a dose-dependent manner in HSC-4 cells (Figure 3B and supplementary material, Figure S3C). Two different siRNAs knocked down PIEZO1 mRNA and protein, but not PIEZO2 mRNA expression (Figure 3C and supplementary material, Figure S3D). Importantly, PIEZO1 knockdown did not change the mRNA levels of YAP transcriptional targets, such as ANKRD1 and CYR61, and YAP/TAZ nuclear localization, indicating that YAP signaling would not be regulated by PIEZO1 in OSCC cells (supplementary material, Figure S3D). Knockdown of PIEZO1 
suppressed the Yoda1-dependent $\mathrm{Ca}^{2+}$ influx (Figure 3D and supplementary material, Figure S3E). Since Yoda1 treatment stimulated ERK1/2 and p38 MAPK activation in human bone marrow-derived mesenchymal stem cells [50], the effect of PIEZO1 on its activation was examined in HSC-4 cells. PIEZO1 knockdown reduced Yoda1-induced ERK1/2 and p38 MAPK phosphorylation, but did not affect the AKT phosphorylation (Figure 3E). Supporting our results, previous report revealed that gain-of-function mutations in PIEZO1 increased $\mathrm{Ca}^{2+}$ concentrations with ERK1/2 activation, indicating that the expression of PIEZO1 channel activates intracellular signaling [51]. These results suggest that PIEZO1 may function as an ion channel and its expression is involved in agonist-dependent ERK1/2 and p38 MAPK activation in OSCC cells.

PIEZO1 expression is required for cell proliferation in oral squamous cell carcinoma cells

As ERK1/2 and p38 MAPK activation is involved in cell proliferation [52,53], the effect of PIEZO1 on cell proliferation was examined in OSCC cells. Knockdown of PIEZO1 decreased the ratio of Ki-67-positive or PCNA-positive cells and cell proliferation capability in HSC-4 cells (Figure 4A and 4B, and supplementary material, Figure S4A), but did not increase the number of cleaved caspase-3-positive cells (supplementary material, Figure S4B). GsMTx-4, a mechanosensitive and stretch-activated ion channel inhibitor and also functions as an inhibitor of PIEZO1 [20,51], decreased cell proliferation (Figure 4C). To further clarify the function of PIEZO1, we generated HSC-4 cells stably expressing PIEZO1 \#1 or \#2 shRNA (Figure 4D). Based on the above results using 
siRNAs, target sequences of PIEZO1 \#1 and \#2 shRNAs were designed to be identical to those of PIEZO1 \#1 and \#2 siRNAs, respectively. In these cells, Yoda1-dependent $\mathrm{Ca}^{2+}$ influx was suppressed, which was consistent with our experiments using siRNAs (Figures 3D and 4E, and supplementary material, Figure S4C). In 3D culture, LATS2 knockdown or YAP ${ }^{5 \mathrm{SA}}$ expression increased the ratio of $\mathrm{Ki}-67$ - or PCNA-positive cells in control cells (Figure 4F and 4G and supplementary material, Figure S4D). Importantly, in HSC4 cells expressing PIEZO1 \#1 or \#2 shRNA, LATS2 knockdown or YAP5SA expression did not change the ratio of Ki-67- or PCNA-positive cells and induced the expression of ANKRD1, but not PIEZO1 (Figure 4F and 4G and supplementary material, Figure S4E). These results suggest that YAP signaling promotes cell proliferation through inducing PIEZO1 expression in 3D-cultured OSCC cells.

\section{PIEZO1 and YAP are expressed in human oral squamous cell carcinoma tissues}

Immunohistochemical analyses were performed to examine PIEZO1 and YAP expression in human OSCC specimens. PIEZO1 expression was detected in 45/45 (100\%) tumor lesions and 25/31 (80.6\%) non-tumor regions (Figure 5A and supplementary material, Figure S5). Based on the intensity of the PIEZO1-positive signal, the samples were divided into three groups: negative, weak and strong (Figure 5A). PIEZO1 was strongly expressed in the cellular cytoplasm in 23/45 (51.1\%) tumor lesions, while a strong expression was noted in 2/31 (6.5\%) non-tumor regions, showing a significant difference $(p<0.0001$; Fisher's exact test) (Figure 5A).

Based on the YAP expression and localization, specimens were divided into three 
groups: negative, nucleus/cytoplasm and nucleus (supplementary material, Figure S6A). When YAP was expressed in nucleus/cytoplasm or nucleus, the results were defined as a YAP-positive case, in which the ratio of YAP-positive cells was more than $30 \%$ in a tumor lesion or a non-tumor region. YAP-positive cases were 43/45 (95.6\%) in tumor lesions, while those were 10/31 (32.3\%) in non-tumor regions (supplementary material, Figure S6B), showing a significant difference $(p<0.0001$; Fisher's exact test). Notably, YAP was hardly detected in nucleus in non-tumor regions. Mice with tongue-specific deletion of MOB kinase activator 1A/B developed tongue carcinoma through endogenous YAP1 hyperactivation, in which YAP1 was localized in nucleus at high frequencies [37]. Therefore, we speculated that YAP might be hyperactivated in tumor lesions of our OSCC specimens. As expected, the YAP-positive expression was frequently detected in tumor lesions with Ki-67 expression but not in non-tumor regions (Figure 5B and supplementary material, Figure S6C), suggesting that hyperactivated YAP might promote cancer cell proliferation. Significant correlations with the PIEZO1 intensity or the YAP expression and localization were not shown for the clinical $\mathrm{T}$ (tumor size) stage, histological grade and lymph node metastasis (Table 1 and 2). It is noteworthy that YAP-positive cases with a strong PIEZO1 expression in tumor lesions were more frequent, but not significantly, than in non-tumor regions in consecutive sections (Figure 5B and supplementary material, Figure S7). Taken together, these results suggest that YAP, which might be hyperactivated in tumor lesion, likely induces PIEZO1 expression, resulting in enhanced OSCC cell proliferation. 


\section{Discussion}

It is generally accepted that the changes in the tumorous extracellular environments, including ECM stiffness and exposure to soluble signals, affect tumor cell behavior [6]. We aimed to identify a molecule, which is activated by extracellular environments, to regulate OSCC tumorigenesis. In the current suspension culture and $3 \mathrm{D}$ culture, OSCC cell proliferation capabilities were reduced. Subsequently, we identified PIEZO1 as a transcriptional target of YAP signaling and showed that an elevated PIEZO1 expression was required for cell proliferation of OSCC cells.

To our knowledge, this is the first report to suggest that PIEZO1 might function as an oncogene-related molecule in OSCC. Similar to our immunohistochemical data, PIEZO1positive signals were detected in the cytoplasm of human prostate carcinoma tissue [23]. In Madin-Darby canine kidney normal epithelial cells, PIEZO1 developed into large cytoplasmic aggregates in the crowded regions with increased cell density [19]. Considering these results, the cytoplasmic localization of PIEZO1 in tumor lesions would correlate with its function, but underlying mechanism is not clear. Cryo-electron microscopy and high-speed atomic force microscopy showed that PIEZO1 structural deformation gates its channel in response to mechanical stress [54], thereby inducing $\mathrm{Ca}^{2+}$ influx [55]. Therefore, the effects of PIEZO1 on OSCC tumorigenesis might depend on PIEZO1 expression level.

In the present study, suspension culture was used to elucidate the hierarchical relationship between YAP and PIEZO1. At present, we do not know why PIEZO1 was not suggested as a down-regulated gene in the microarray data (supplementary material, 
Table S4).

Recently, it has been reported that PIEZO1 could act as an upstream of YAP in the

developmental stages, such as human neural stem cell differentiation and zebrafish heart development $[20,26]$. In this study, PIEZO1 knockdown did not affect the expression of YAP transcriptional targets and YAP/TAZ nuclear localization. In addition, gain-offunction experiments and ChIP data demonstrated that YAP signaling promotes PIEZO1 expression in OSCC cells. Although the hierarchical relationship between YAP signaling and PIEZO1 may depend on cell context, it is noteworthy that these molecules could be a common mechanotransduction mechanism between organ formation by stem cells and tumor formation by cancer cells.

The current immunohistochemical data demonstrated YAP localization in nucleus and/or cytoplasm in tumor lesions with an increased Ki-67-positive signal, suggesting that YAP signaling might be activated with enhanced OSCC cellular growth. But underlying mechanism to activate YAP signaling is unclear. In non-tumor regions, we also confirmed a low frequency of YAP-positive expression in nucleus/cytoplasm with a decreased Ki-67-positive signal (see supplementary material, Figure S6C), but its precise function in the regions remains unclear. As the Hippo pathway regulates cell differentiation as well as cell proliferation [45], cancer therapy targeting for components of the Hippo pathway might cause side effects in the non-tumor region, at least in OSCC. If the transcriptional targets mediating the Hippo pathway in regulating cellular growth may function in tumor lesion specifically, its gene could be a molecular target for novel cancer therapy. 
Some genetic mutations of cancer-related genes, which are responsible for tumorigenesis and might be molecular targets of anti-tumor therapy for OSCC, have been reported [56]. Recent report also demonstrated that the resistance to phosphoinositide-3kinase (PI3K)- $\alpha$ inhibitors for head and neck SCC bearing PIK3 catalytic subunit alpha (PIK3CA) mutations and amplification [57], indicating that the therapeutic options for patients with head and neck SCC are limited. Another molecular mechanism could be involved in OSCC tumorigenesis. We previously demonstrated that TRPV4, a $\mathrm{Ca}^{2+}$ channel, enhances OSCC cell proliferation through CaMKII-AKT activation [9]. Given the present and previous findings, it is intriguing to speculate that $\mathrm{Ca}^{2+}$ influx-dependent cellular growth through PIEZO1 or TRPV4 may be a potential target for anti-tumor therapy.

In summary, we found that YAP, which is activated in OSCC tumor lesion, promotes cancer cell proliferation. We also demonstrated that YAP signaling induces PIEZO1 expression to regulate OSCC cellular growth positively. Taken together, these results suggest that the YAP/PIEZO1 axis contributes to OSCC cell growth. 


\section{Acknowledgments}

The authors thank Drs. T. Harada, M. Nishio, A. Suzuki, I. Imajo and H. Wada for advices and searching clinical information in this research. The authors also thank the Research Support Center, Graduate School of Medical Sciences, Kyushu University. This work was supported by JSPS KAKENHI Grants to K. H. (2017-2018) (17K17103), (20192020) (19K18966), S. F. (2020-2022) (20K09906) and T. K. (2020-2022) (20K10096), and Takeda Science Foundation and The Shin-Nihon Foundation of Advanced Medical Research to S.F.

\section{Author contributions}

K. H. carried out experiments, analyzed data and co-wrote the manuscript. S. F. carried out experiments, conceived and wrote the manuscript. S. M. and A. K. performed ChIP experiments. Y. T. analyzed immunohistochemical data. T. K. carried out data interpretation and co-wrote the manuscript. All authors were involved in writing the paper and had final approval of the submitted and published versions. 


\section{Supporting Information}

\section{Supplementary figure legends}

Figure S1. The effect of YAP/TAZ signaling on OSCC cells.

Figure S2. YAP signaling regulates PIEZO1 expression in OSCC cells.

Figure S3. The effect of PIEZO1 on YAP/TAZ signaling.

Figure S4. PIEZO1 regulates cell proliferation of OSCC cells.

Figure S5. PIEZO1 is expressed in human oral squamous cell carcinoma tissues.

Figure S6. YAP is expressed in human oral squamous cell carcinoma tissues.

Figure S7. Co-expression of PIEZO1, YAP, Ki-67 and PCNA in human oral squamous cell carcinoma tissues.

Table S1. List of siRNA sequence used in this study.

Table S2. List of quantitative RT-PCR primer used in this study.

Table S3. Gene enrichment analysis in HSC-4 cells.

Table S4. List of gene from microarray analysis data of HSC-4 cells cultured in 2D culture and suspension culture. 


\section{References}

1. Warnakulasuriya S. Global epidemiology of oral and oropharyngeal cancer. Oral Oncol 2009; 45: 309-316.

2. Bray F, Ferlay J, Soerjomataram I, et al. Global cancer statistics 2018: GLOBOCAN estimates of incidence and mortality worldwide for 36 cancers in 185 countries. CA Cancer J Clin 2018; 68: 394-424.

3. Bloebaum M, Poort L, Böckmann R, et al. Survival after curative surgical treatment for primary oral squamous cell carcinoma. J Craniomaxillofac Surg 2014; 42: 1572-1576.

4. Anderson CR, Sisson K, Moncrieff M. A meta-analysis of margin size and local recurrence in oral squamous cell carcinoma. Oral Oncol 2015; 51: 464-469.

5. Wang B, Zhang S, Yue K, et al. The recurrence and survival of oral squamous cell carcinoma: a report of 275 cases. Chin J Cancer 2013; 32: 614-618.

6. Fares J, Fares MY, Khachfe HH, et al. Molecular principles of metastasis: a hallmark of cancer revisited. Signal Transduct Target Ther 2020; 5: 28.

7. Levental KR, Yu H, Kass L, et al. Matrix crosslinking forces tumor progression by enhancing integrin signaling. Cell 2009; 139: 891-906.

8. Dou C, Liu Z, Tu K, et al. P300 Acetyltransferase Mediates Stiffness-Induced Activation of Hepatic Stellate Cells Into Tumor-Promoting Myofibroblasts. Gastroenterology 2018; 154: 2209-2221.

9. Fujii S, Tajiri Y, Hasegawa K, et al. The TRPV4-AKT axis promotes oral squamous cell carcinoma cell proliferation via CaMKII activation. Lab Invest 2020; 100: 311-323.

10. Saucedo LJ, Edgar BA. Filling out the Hippo pathway. Nat Rev Mol Cell Biol 2007; 8: 613-621.

11. Dupont $\mathrm{S}$, Morsut $\mathrm{L}$, Aragona $\mathrm{M}$, et al. Role of YAP/TAZ in mechanotransduction. Nature 2011; 474: 179-183.

12. Matsumoto S, Fujii S, Sato A, et al. A combination of Wnt and growth factor signaling induces Arl4c expression to form epithelial tubular structures. EMBO J 2014; 33: 702-718.

13. Meng Z, Qiu Y, Lin KC, et al. RAP2 mediates mechanoresponses of the Hippo pathway. Nature 2018; 560: 655-660. 
14. Hiemer SE, Zhang L, Kartha VK, et al. A YAP/TAZ-Regulated Molecular Signature Is Associated with Oral Squamous Cell Carcinoma. Mol Cancer Res 2015; 13: 957-968.

15. $\mathrm{Xu} \mathrm{MZ,} \mathrm{Yao} \mathrm{TJ,} \mathrm{Lee} \mathrm{NP,} \mathrm{et} \mathrm{al.} \mathrm{Yes-associated} \mathrm{protein} \mathrm{is} \mathrm{an} \mathrm{independent}$ prognostic marker in hepatocellular carcinoma. Cancer 2009; 115: 4576-4585.

16. Kim HM, Jung WH, Koo JS. Expression of Yes-associated protein (YAP) in metastatic breast cancer. Int J Clin Exp Pathol 2015; 8: 11248-11257.

17. Zhang X, George J, Deb S, et al. The Hippo pathway transcriptional co-activator, YAP, is an ovarian cancer oncogene. Oncogene 2011; 30: 2810-2822.

18. Coste B, Mathur J, Schmidt M, et al. Piezo1 and Piezo2 are essential components of distinct mechanically activated cation channels. Science 2010; 330: 55-60.

19. Gudipaty SA, Lindblom J, Loftus PD, et al. Mechanical stretch triggers rapid epithelial cell division through Piezo1. Nature 2017; 543: 118-121.

20. Pathak MM, Nourse JL, Tran T, et al. Stretch-activated ion channel Piezo1 directs lineage choice in human neural stem cells. Proc Natl Acad Sci U S A 2014; 111: $16148-16153$.

21. Li J, Hou B, Tumova S, et al. Piezo1 integration of vascular architecture with physiological force. Nature 2014; 515: 279-282.

22. Yang XN, Lu YP, Liu JJ, et al. Piezo1 is as a novel trefoil factor family 1 binding protein that promotes gastric cancer cell mobility in vitro. Dig Dis Sci 2014; 59: 1428-1435.

23. Han Y, Liu C, Zhang D, et al. Mechanosensitive ion channel Piezo1 promotes prostate cancer development through the activation of the Akt/mTOR pathway and acceleration of cell cycle. Int J Oncol 2019; 55: 629-644.

24. McHugh BJ, Murdoch A, Haslett C, et al. Loss of the integrin-activating transmembrane protein Fam38A (Piezo1) promotes a switch to a reduced integrindependent mode of cell migration. PLoS One 2012; 7: e40346.

25. Huang Z, Sun Z, Zhang X, et al. Loss of stretch-activated channels, PIEZOs, accelerates non-small cell lung cancer progression and cell migration. Biosci Rep $2019 ; 39$.

26. Duchemin AL, Vignes H, Vermot J. Mechanically activated piezo channels modulate outflow tract valve development through the Yap1 and Klf2-Notch signaling axis. Elife 2019; 8: eLife.44706. 
27. Matsuo K, Ishibashi Y, Kobayashi I, et al. New human oral squamous carcinoma cell line and its tumorigenic subline producing granulocyte colony-stimulating factor. Jpn J Cancer Res 1994; 85: 1257-1262.

28. Fujii S, Matsumoto S, Nojima S, et al. Arl4c expression in colorectal and lung cancers promotes tumorigenesis and may represent a novel therapeutic target. Oncogene 2015; 34: 4834-4844.

29. Mikami Y, Fujii S, Nagata K, et al. GLI-mediated Keratin 17 expression promotes tumor cell growth through the anti-apoptotic function in oral squamous cell carcinomas. J Cancer Res Clin Oncol 2017; 143: 1381-1393.

30. Miyoshi H, Blömer U, Takahashi M, et al. Development of a self-inactivating lentivirus vector. J Virol 1998; 72: 8150-8157.

31. Fujii S, Shinjo K, Matsumoto S, et al. Epigenetic upregulation of ARL4C, due to DNA hypomethylation in the 3 '-untranslated region, promotes tumorigenesis of lung squamous cell carcinoma. Oncotarget 2016; 7: 81571-81587.

32. Matsumoto S, Kurimoto T, Taketo MM, et al. The WNT/MYB pathway suppresses KIT expression to control the timing of salivary proacinar differentiation and duct formation. Development 2016; 143: 2311-2324.

33. Fujii S, Nagata $\mathrm{K}$, Matsumoto $\mathrm{S}$, et al. Wnt/ $\beta$-catenin signaling, which is activated in odontomas, reduces Sema3A expression to regulate odontogenic epithelial cell proliferation and tooth germ development. Sci Rep 2019; 9: 4257.

34. Takata T, Slootweg PJ. Tumours of the oral cavity and mobile tongue. Solan P, Gale N, Hunter $\mathrm{K}$, et al. Malignant surface epithelial tumours. In WHO classification of head and neck tumours, (4th edn), El-Naggar AK,Chan JKC, Grandis JR, et al. (eds). IARC: Lyon, 2017; 108-111

35. O'Sullivan B. Head and Neck Tumours. In TNM Classification of Malignant Tumours, (8th edn), Brierley JD, Gospodarowicz MK, Wittekind C (eds). John Wiley \& Sons, Ltd: Hoboken, 2017; 17-21.

36. Wang $\mathrm{Z}, \mathrm{Wu} \mathrm{Y}$, Wang $\mathrm{H}$, et al. Interplay of mevalonate and Hippo pathways regulates RHAMM transcription via YAP to modulate breast cancer cell motility. Proc Natl Acad Sci U S A 2014; 111: E89-98.

37. Omori H, Nishio M, Masuda M, et al. YAP1 is a potent driver of the onset and progression of oral squamous cell carcinoma. Sci Adv 2020; 6: eaay3324.

38. Yabuta N, Mukai S, Okada N, et al. The tumor suppressor Lats2 is pivotal in 
Aurora A and Aurora B signaling during mitosis. Cell cycle 2011; 10: 2724-2736.

39. Oku Y, Nishiya N, Tazawa T, et al. Augmentation of the therapeutic efficacy of WEE1 kinase inhibitor AZD1775 by inhibiting the YAP-E2F1-DNA damage response pathway axis. FEBS Open Bio 2018; 8: 1001-1012.

40. Fesquet D, De Bettignies G, Bellis M, et al. Binding of Kif23-iso1/CHO1 to 143-3 is regulated by sequential phosphorylations at two LATS kinase consensus sites. PLos One 2015; 10: e0117857.

41. Zanconato F, Forcato M, Battilana G, et al. Genome-wide association between YAP/TAZ/TEAD and AP-1 at enhancers drives oncogenic growth. Nat Cell Biol 2015; 17: 1218-1227.

42. Eisinger-Mathason TS, Mucaj V, Biju KM, et al. Deregulation of the Hippo pathway in soft-tissue sarcoma promotes FOXM1 expression and tumorigenesis. Proc Natl Acad Sci U S A 2015; 112: E3402-3411.

43. Li Z, Razavi P, Li Q, et al. Loss of the FAT1 Tumor Suppressor Promotes Resistance to CDK4/6 Inhibitors via the Hippo Pathway. Cancer cell 2018; 34: 893-905.

44. Zhang J, Ji JY, Yu M, et al. YAP-dependent induction of amphiregulin identifies a non-cell-autonomous component of the Hippo pathway. Nat Cell Biol 2009; 11: 1444-1450.

45. Goto $\mathrm{H}$, Nishio $\mathrm{M}$, To $\mathrm{Y}$, et al. Loss of Mobla/b in mice results in chondrodysplasia due to YAP1/TAZ-TEAD-dependent repression of SOX9. Development 2018; 145. dev159244.

46. Varelas X. The Hippo pathway effectors TAZ and YAP in development, homeostasis and disease. Development 2014; 141: 1614-1626.

47. Stein C, Bardet AF, Roma G, et al. YAP1 Exerts Its Transcriptional Control via TEAD-Mediated Activation of Enhancers. PLoS Genet 2015; 11: e1005465.

48. Syeda R, Xu J, Dubin AE, et al. Chemical activation of the mechanotransduction channel Piezo1. Elife 2015; 4. eLife.07369.

49. Blythe NM, Muraki K, Ludlow MJ, et al. Mechanically activated Piezo1 channels of cardiac fibroblasts stimulate p38 mitogen-activated protein kinase activity and interleukin-6 secretion. J Biol Chem 2019; 294: 17395-17408.

50. Sugimoto A, Miyazaki A, Kawarabayashi K, et al. Piezo type mechanosensitive ion channel component 1 functions as a regulator of the cell fate determination of 
mesenchymal stem cells. Sci Rep 2017; 7: 17696.

51. Andolfo I, Rosato BE, Manna F, et al. Gain-of-function mutations in PIEZO1 directly impair hepatic iron metabolism via the inhibition of the BMP/SMADs pathway. Am J Jematol 2020; 95: 188-197.

52. Zhang W, Liu HT. MAPK signal pathways in the regulation of cell proliferation in mammalian cells. Cell Res 2002; 12: 9-18.

53. Cuadrado A, Nebreda AR. Mechanisms and functions of p38 MAPK signalling. Biochem J 2010; 429: 403-417.

54. Lin YC, Guo YR, Miyagi A, et al. Force-induced conformational changes in PIEZO1. Nature 2019; 573: 230-234.

55. Miyamoto T, Mochizuki T, Nakagomi H, et al. Functional role for Piezo1 in stretch-evoked $\mathrm{Ca}^{2+}$ influx and ATP release in urothelial cell cultures. $J$ Biol Chem 2014; 289: 16565-16575.

56. Cancer Genome Atlas Network. Comprehensive genomic characterization of head and neck squamous cell carcinomas. Nature 2015; 517: 576-582.

57. Elkabets M, Pazarentzos E, Juric D, et al. AXL mediates resistance to PI3K $\alpha$ inhibition by activating the EGFR/PKC/mTOR axis in head and neck and esophageal squamous cell carcinomas. Cancer cell 2015; 27: 533-546. 
Table 1. Relationship between the PIEZO1 intensity and clinicopathological characteristics of OSCC cases.

\begin{tabular}{|c|c|c|c|c|c|}
\hline & & \multicolumn{3}{|c|}{ PIEZO1 expression } & \multirow[t]{2}{*}{$P$ value } \\
\hline & & Negative & Weak & strong & \\
\hline \multicolumn{6}{|c|}{ Clinical T stage } \\
\hline $\mathrm{T} 1$ & 7 & 0 & 3 & 4 & \\
\hline $\mathrm{T} 2$ & 20 & 0 & 13 & 7 & \\
\hline $\mathrm{T} 3$ & 7 & 0 & 4 & 3 & \\
\hline $\mathrm{T} 4$ & 11 & 0 & 2 & 9 & N.S. ${ }^{\text {a }}$ \\
\hline \multicolumn{6}{|c|}{ Histologic grade } \\
\hline Well & 25 & 0 & 14 & 11 & \\
\hline Moderately & 16 & 0 & 7 & 9 & \\
\hline Poorly & 4 & 0 & 1 & 3 & N.S. ${ }^{\text {a }}$ \\
\hline \multicolumn{6}{|l|}{$\begin{array}{l}\text { Lymph node } \\
\text { metastasis }\end{array}$} \\
\hline Positive & 11 & 0 & 4 & 7 & \\
\hline Negative & 34 & 0 & 18 & 16 & N.S. ${ }^{a}$ \\
\hline
\end{tabular}

\begin{abstract}
Abbreviations: Clinical $\mathrm{T}$ stage; $T 1$ tumor $2 \mathrm{~cm}$ or less in greatest dimension and $5 \mathrm{~mm}$ or less depth of invasion, $T 2$ tumor $2 \mathrm{~cm}$ or less in greatest dimension and more than 5 $\mathrm{mm}$ but no more than $10 \mathrm{~mm}$ depth of invasion or tumor more than $2 \mathrm{~cm}$ but not more than $4 \mathrm{~cm}$ in greatest dimension and depth of invasion no more than $10 \mathrm{~mm}, T 3$ tumor more than $2 \mathrm{~cm}$ but not more than $4 \mathrm{~cm}$ in greatest dimension and depth of invasion more than $10 \mathrm{~mm}$ or tumor more than $4 \mathrm{~cm}$ in greatest dimension and not more than $10 \mathrm{~mm}$ depth of invasion, T4 (T4a and T4b): T4a (lip) tumor invades through cortical bone, inferior alveolar nerve, floor of mouth, or skin (of the chin or nose), T4a (oral cavity) tumor more than $4 \mathrm{~cm}$ in greatest dimension and more than $10 \mathrm{~mm}$ depth of invasion or
\end{abstract}


tumor invades through the cortical bone of the mandible or maxilla or involves the maxillary sinus, or invades the skin of the face, T4b (lip and oral cavity) tumor invades masticator space, pterygoid plates, or skull base, or encases internal carotid artery.

Histologic grade (WHO); Grade 1 well differentiated, Grade 2 moderately differentiated, Grade 3 poorly differentiated.

a Statistical analyses were performed by Fisher's exact test. N.S.: not significant. 
Table 2. Relationship between the YAP expression and localization, and clinicopathological characteristics of OSCC cases.

\begin{tabular}{|c|c|c|c|c|c|}
\hline & & \multicolumn{3}{|c|}{ YAP expression and localization } & \multirow[t]{2}{*}{$P$ value } \\
\hline & & Negative & Nucleus/cytoplasm & Nucleus & \\
\hline \multicolumn{6}{|c|}{ Clinical T stage } \\
\hline $\mathrm{T} 1$ & 7 & 0 & 3 & 4 & \\
\hline $\mathrm{T} 2$ & 20 & 1 & 5 & 14 & \\
\hline $\mathrm{T} 3$ & 7 & 1 & 2 & 4 & \\
\hline $\mathrm{T} 4$ & 11 & 0 & 3 & 8 & N.S. ${ }^{a}$ \\
\hline \multicolumn{6}{|c|}{ Histologic grade } \\
\hline Well & 25 & 1 & 9 & 15 & \\
\hline Moderately & 16 & 1 & 4 & 11 & \\
\hline Poorly & 4 & 0 & 0 & 4 & N.S. ${ }^{a}$ \\
\hline \multicolumn{6}{|l|}{$\begin{array}{l}\text { Lymph node } \\
\text { metastasis }\end{array}$} \\
\hline Positive & 11 & 2 & 4 & 5 & \\
\hline Negative & 34 & 0 & 9 & 25 & N.S. ${ }^{a}$ \\
\hline
\end{tabular}

Abbreviations are same in the Table 1.

a Statistical analyses were performed by Fisher's exact test. N.S.: not significant. 


\section{Figure Legends}

Figure 1. Proliferative capabilities of oral squamous cell carcinoma cells in the threedimensional and suspension cultures.

(A) HSC-4 cells $\left(1 \times 10^{5}\right.$ cells $)$ and HSC-3 cells $\left(1 \times 10^{5}\right.$ cells $)$ were seeded in $2 \mathrm{D}, 3 \mathrm{D}$ (collagen or Matrigel) or suspension culture in the presence of 10\% FBS for $48 \mathrm{~h}$, and relative cell numbers were quantified using the CyQUANT cell proliferation assay. RFU: relative fluorescence units. (B) Gene enrichment analysis of down-regulated genes in suspension culture comparing with 2D culture for gene ontology biological processes was carried out. (C) HSC-4 cells were cultured in 2D or suspension culture for the indicated numbers of hours. Cell lysates were probed with anti-YAP/TAZ and anti- $\beta$-actin antibodies. Molecular weight size was noted referring to molecular weight marker. (D) ANKRD1 and CYR61 mRNA levels in HSC-4 cells cultured in 2D or suspension culture for $24 \mathrm{~h}$ were measured by quantitative RT-PCR. Relative levels of ANKRD1 and CYR61 mRNA expression were normalized to GAPDH and expressed as fold-changes compared with expression in 2D cells. (E) HSC-4 cells were transfected with control or LATS2 siRNA for $48 \mathrm{~h}$, and then cells were cultured in 2D or suspension culture for $24 \mathrm{~h}$. ANKRD1 and CYR61 mRNA levels were measured by quantitative RT-PCR. Relative levels of $A N K R D 1$ and CYR61 mRNA expression were normalized to GAPDH and expressed as fold-changes compared with levels in 2D control cells. Results are shown as means \pm s.d. of three independent experiments. $* P<0.05, * * P<0.01$. n.s.: not significant.

Figure 2. PIEZO1 is a transcriptional target of YAP signaling in oral squamous cell 
carcinoma cells.

(A) HSC-4 cells were cultured in 2D (UT), 2D (Coat) or 3D (collagen) culture for $24 \mathrm{~h}$. PIEZO1 mRNA levels were measured by quantitative RT-PCR. Relative levels of PIEZO1 mRNA expression were normalized to GAPDH and expressed as fold-changes compared with expression in 2D (UT) cells. Cell lysates were probed with anti-PIEZO1 and anti- $\beta$-actin antibodies. Molecular weight size was noted referring to molecular weight marker. (B) HSC-4 cells were cultured in 2D or suspension culture for the indicated numbers of hours. PIEZO1 mRNA levels were measured by quantitative RTPCR. Relative levels of PIEZO1 mRNA expression were normalized to GAPDH and expressed as fold-changes compared with expression in 2D cells. Cell lysates were probed with anti-PIEZO1 and anti- $\beta$-actin antibodies. Molecular weight size was noted referring to molecular weight marker. (C) HSC-4 cells were transfected with control or LATS2 siRNA for $48 \mathrm{~h}$, and then cells were cultured in 2D or suspension culture for $24 \mathrm{~h}$. PIEZO1 mRNA levels were measured by quantitative RT-PCR. Relative levels of PIEZO1 mRNA expression were normalized to GAPDH and expressed as fold-changes compared with expression in 2D control cells. (D) HSC-4 cells were transfected with control or two different YAP/TAZ siRNAs for $48 \mathrm{~h}$, and then cells were cultured in 2D culture for $3 \mathrm{~h}$. PIEZO1 mRNA levels were measured by quantitative RT-PCR. Relative PIEZO1 mRNA levels were normalized by GAPDH and expressed as fold-changes compared with levels in control siRNA transfected cells. (E) HSC-4 cells expressing mock or YAP5SA were cultured in 2D or suspension culture for $24 \mathrm{~h}$ and PIEZO1 mRNA levels were measured by quantitative RT-PCR (left panel). Relative levels of PIEZO1 mRNA expression were 
normalized to GAPDH and expressed as fold-changes compared with expression in 2D control cells. Cell lysates were probed with anti-PIEZO1, anti-YAP/TAZ and anti- $\beta$-actin antibodies (right panel). Molecular weight size was noted referring to molecular weight marker. Arrowhead indicates non-specific bands. (F) Chromatin from HSC-4 cells expressing mock or YAP5SA was immunoprecipitated with anti-control IgG or antiTEAD4 and anti-FLAG antibodies, and the precipitates were analyzed by PCR for TEAD4 binding site 1, site 2 or site 3 of the PIEZO1 gene. White box indicates TEAD4 binding motif and site 3 has two TEAD4 binding motifs. Results are shown as means \pm s.d. of three independent experiments. ${ }^{*} P<0.05$, ${ }^{*} P<0.01$. n.s.: not significant.

Figure 3. PIEZO1 expression is involved in agonist-induced ERK1/2 and p38 MAPK activation.

(A) PIEZO1 mRNA levels were measured in OSCC cell lines (HSC-4, HSC-3, HSC-2, SAS, MISK81-5 and sMISK cells) by quantitative RT-PCR. Relative levels of PIEZO1 mRNA expression were normalized to GAPDH and expressed as fold-changes compared with expression in control MKN-45 cells. (B) HSC-4 cells were pre-loaded with Fluo-4$\mathrm{AM}$ and were exposed without or with 1 and $10 \mu \mathrm{M}$ Yoda1. Then, intracellular $\mathrm{Ca}^{2+}$ influx at $160 \mathrm{sec}$ was measured. RFU: relative fluorescence units. (C) HSC-4 cells were transfected with control or two different PIEZO1 siRNAs for $48 \mathrm{~h}$, and PIEZO1 mRNA levels were measured by quantitative RT-PCR. Relative PIEZO1 mRNA levels were normalized by GAPDH and expressed as fold-changes compared with levels in control siRNA transfected cells. Cell lysates were probed with anti-PIEZO1 and anti- $\beta$-actin 
antibodies. Molecular weight size was noted referring to molecular weight marker. (D) HSC-4 cells transfected with control or two different PIEZO1 siRNAs were pre-loaded with Fluo-4-AM and were exposed without or with $10 \mu \mathrm{M}$ Yoda1. Then, intracellular $\mathrm{Ca}^{2+}$ influx at $160 \mathrm{sec}$ was measured. RFU: relative fluorescence units. (E) HSC-4 cells were transfected with control or two different PIEZO1 siRNAs, and treated without or with $10 \mu \mathrm{M}$ Yoda1 for $5 \mathrm{~min}$. Cell lysates were probed with anti-phospho-ERK1/2, antiERK1/2, anti-phospho-p38, anti-p38, anti-phospho-AKT and anti-pan-AKT antibodies. Molecular weight size was noted referring to molecular weight marker. Results are shown as means \pm s.d. of three independent experiments. ${ }^{*} P<0.05, * * P<0.01$. n.s.: not significant.

Figure 4. PIEZO1 expression is required for cell proliferation in oral squamous cell carcinoma cells.

(A) HSC-4 cells were transfected with control or PIEZO1 \#1 siRNA for $48 \mathrm{~h}$. The cells were stained with anti-Ki-67 antibody and Hoechst 33342, and then Ki-67-positive cells and Hoechst 33342-stained cells were counted, respectively. Results are expressed as the percentage of Ki-67-positive cells compared with total Hoechst 33342-stained cells. (B) HSC-4 cells were transfected with control or two different PIEZO1 siRNAs. The cells were cultured in the presence of 5\% FBS for the indicated numbers of days, and cell numbers were counted. (C) HSC-4 cells were cultured without or with $100 \mu \mathrm{M}$ GsMTx4 in the absence of FBS for $48 \mathrm{~h}$, and relative cell numbers were quantified using the CyQUANT cell proliferation assay. RFU: relative fluorescence units. (D) PIEZO1 
mRNA levels in HSC-4 cells expressing control or PIEZO1 \#1 and \#2 shRNAs were measured by quantitative RT-PCR. Relative levels of PIEZO1 mRNA expression were normalized to GAPDH and expressed as fold-changes compared with expression in control cells. Lysates of HSC-4 cells expressing control or PIEZO1 \#1 and \#2 shRNAs were probed with anti-PIEZO1 and anti- $\beta$-actin antibodies. Molecular weight size was noted referring to molecular weight marker. (E) HSC-4 cells expressing control or PIEZO1 \#1 and \#2 shRNAs were pre-loaded with Fluo-4-AM and were exposed without or with $10 \mu \mathrm{M}$ Yoda1. Then, intracellular $\mathrm{Ca}^{2+}$ influx at $160 \mathrm{sec}$ was measured. RFU: relative fluorescence units. (F) HSC-4 cells expressing control or PIEZO1 \#1 and \#2 shRNAs were transfected with control or LATS2 siRNA for $48 \mathrm{~h}$, and then cells were cultured for 4 days in the 3D Matrigel. The cells were stained with anti-Ki-67 antibody and Hoechst 33342, and then Ki-67-positive cells and Hoechst 33342-stained cells were counted, respectively. Results are expressed as the percentage of Ki-67-positively stained cells compared with total Hoechst 33342-stained cells $(n=13,469)$. (G) HSC-4 cells expressing control or PIEZO1 \#1 and \#2 shRNAs were transfected with mock or YAP ${ }^{5 S A}$, and then cells were cultured for 4 days in the 3D Matrigel. The cells were stained with anti-Ki-67 antibody and Hoechst 33342, and then Ki-67-positive cells and Hoechst 33342-stained cells were counted, respectively. Results are expressed as the percentage of Ki-67-positively stained cells compared with total Hoechst 33342-stained cells ( $n=$ 5,603). Results are shown as means \pm s.d. of three independent experiments. ${ }^{*} P<0.05$, $* * P<0.01$. Scale bars, $100 \mu \mathrm{m}$. 
Figure 5. PIEZO1 and YAP are expressed in human oral squamous cell carcinoma tissues. (A) Oral squamous cell carcinoma tissues $(n=45)$ were stained with anti-PIEZO1 antibody and hematoxylin. PIEZO1-positive signal intensity was classified as follows: negative; weak; strong. Percentages of three groups based on PIEZO1-positive signal intensity in the non-tumor regions and the tumor lesions are shown in the panel. Boxes show enlarged images. (B) Oral squamous cell carcinoma tissues $(n=45)$ were stained with anti-PIEZO1, anti-YAP and anti-Ki-67 antibodies, and hematoxylin. Solid boxes show enlarged images. Numbers of cases with YAP-positive in non-tumor region or tumor lesion, and PIEZO1 intensity strong or negative/weak was shown. Scale bars, 500 $\mu \mathrm{m}$ (A), $100 \mu \mathrm{m}$ (B). 
A



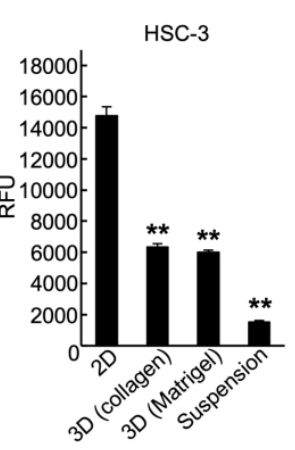

B

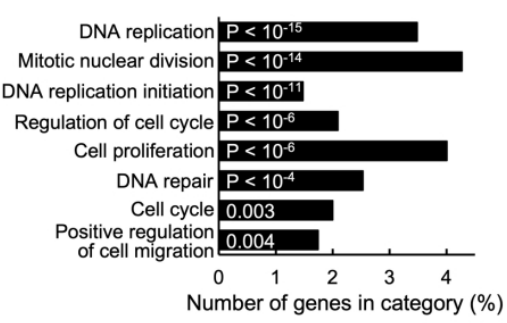

C

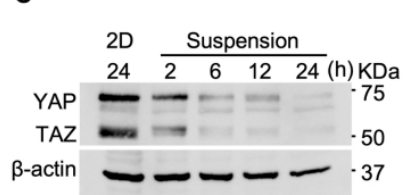

D
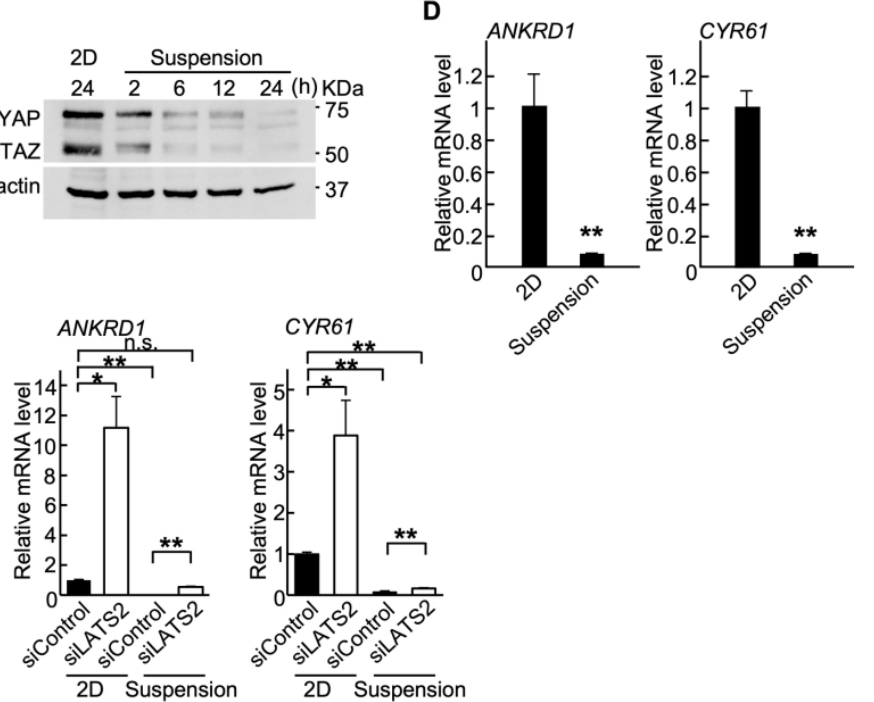

Figure 1. Proliferative capabilities of oral squamous cell carcinoma cells in the three-dimensional and suspension cultures.

$190 \times 254 \mathrm{~mm}(300 \times 300 \mathrm{DPI})$ 


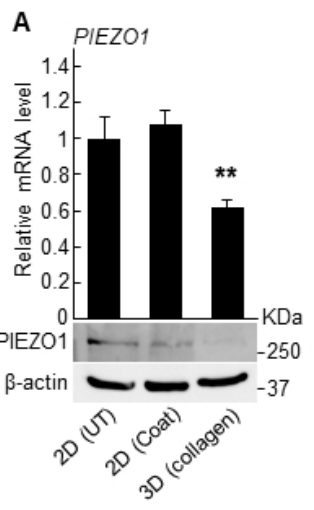

D

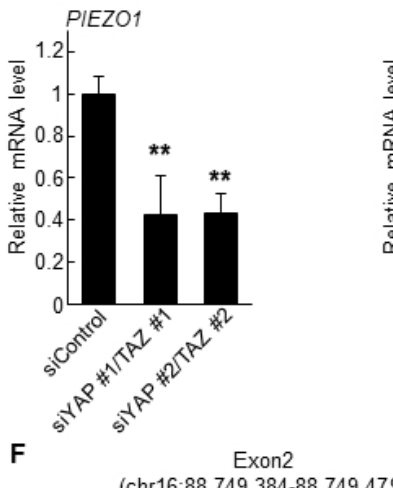

B
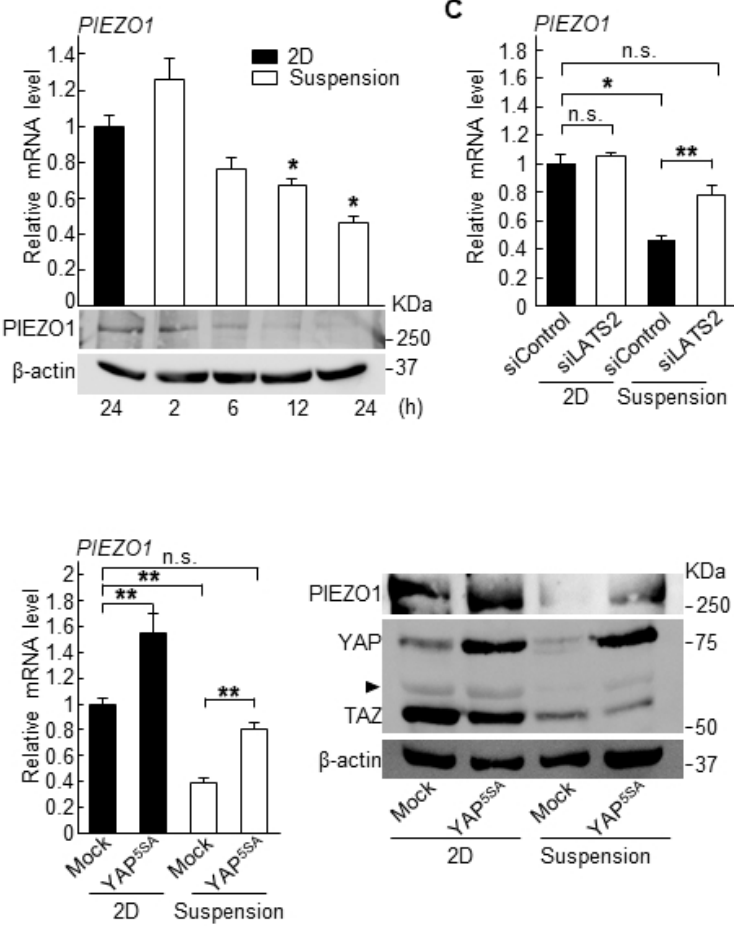

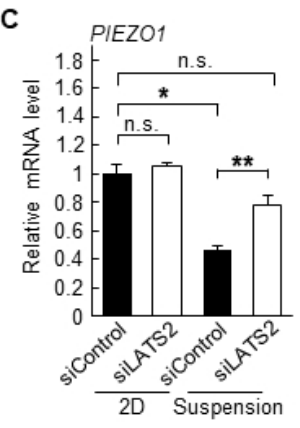

C

Figure 2. PIEZO1 is a transcriptional target of YAP signaling in oral squamous cell carcinoma cells. $190 \times 254 \mathrm{~mm}(96 \times 96 \mathrm{DPI})$ 
A

AIEZO1

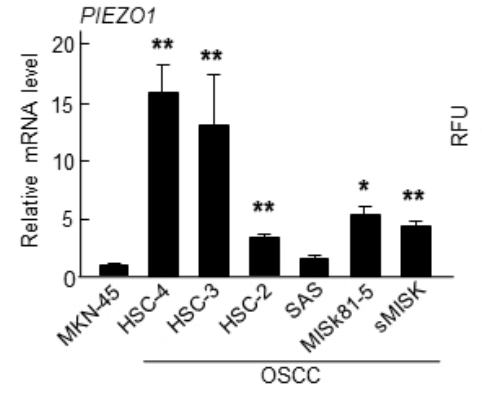

B

C

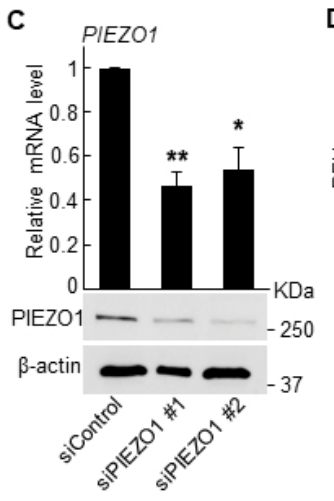

D

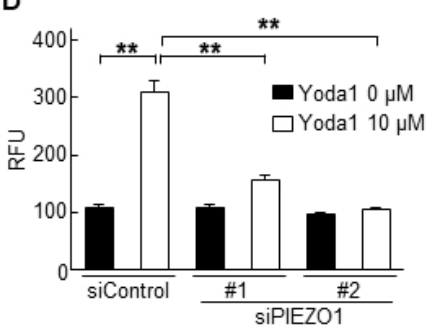

E

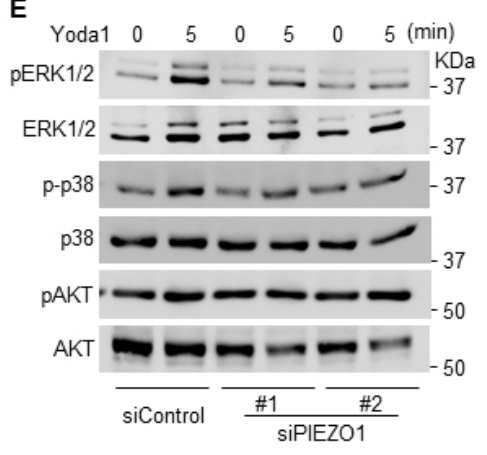

Figure 3. PIEZO1 expression is involved in agonist-induced ERK1/2 and p38 MAPK activation.

$$
190 \times 254 \mathrm{~mm}(96 \times 96 \mathrm{DPI})
$$


A
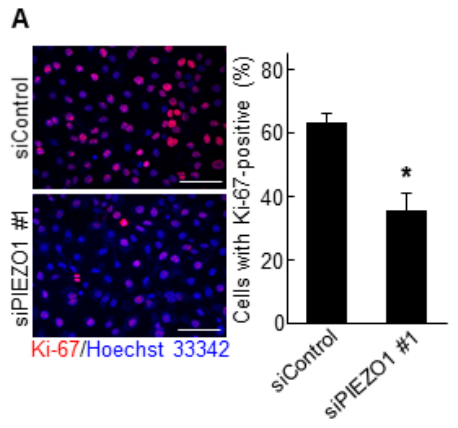

C
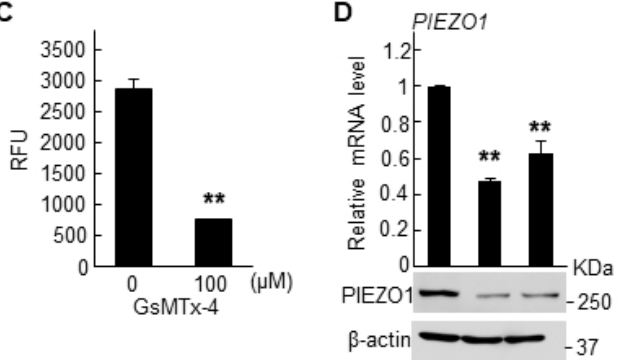

-actin - -37

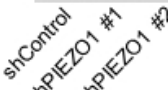

$\mathbf{F}$
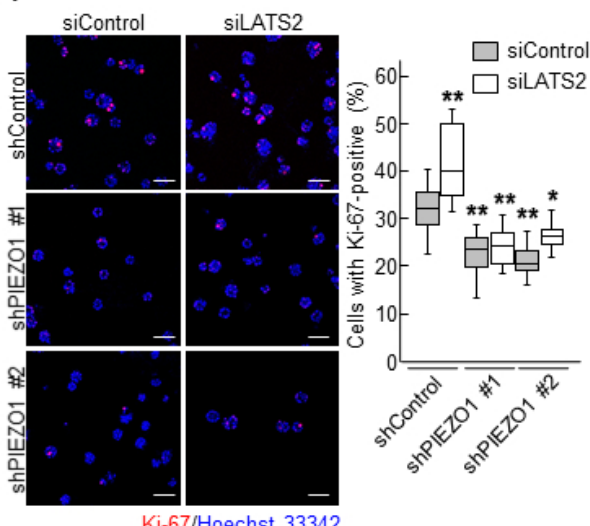

G



Figure 4. PIEZO1 expression is required for cell proliferation in oral squamous cell carcinoma cells. $190 \times 254 \mathrm{~mm}(96 \times 96 \mathrm{DPI})$ 
A

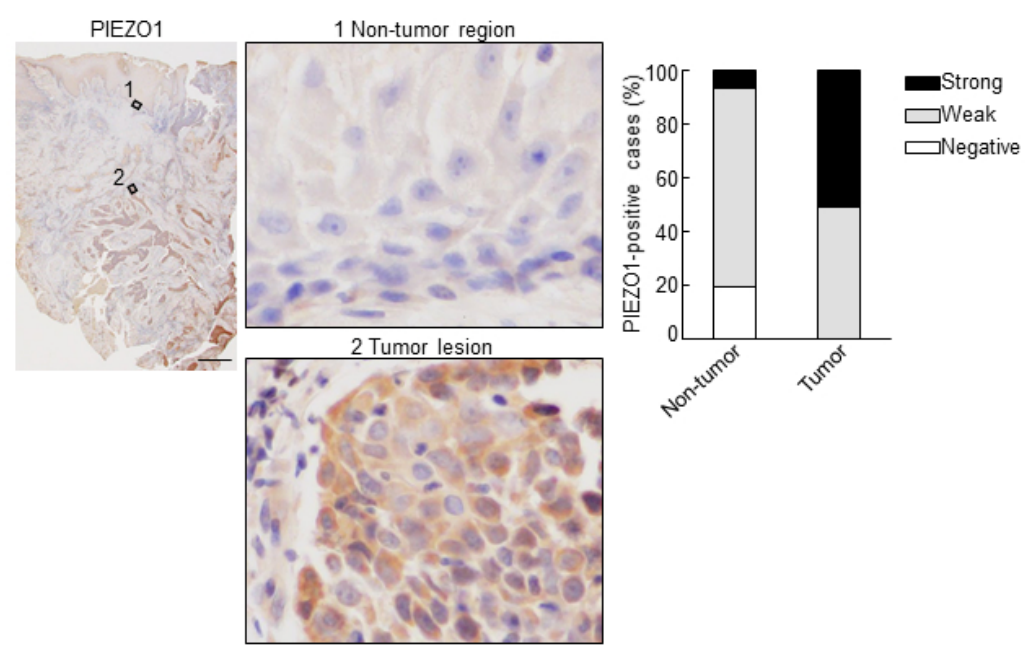

B

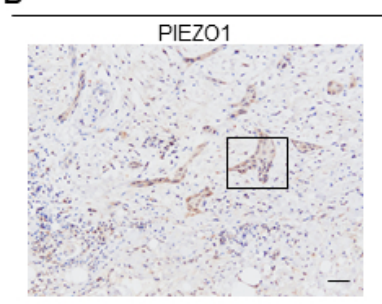

OSCC: Tumor lesion


Figure 5. PIEZO1 and YAP are expressed in human oral squamous cell carcinoma tissues.

$190 \times 254 \mathrm{~mm}(96 \times 96 \mathrm{DPI})$ 


\title{
Supporting Information
}

\section{YAP signaling induces PIEZO1 expression to promote oral squamous cell carcinoma cell proliferation}

\author{
Kana Hasegawa $^{1}$, Shinsuke Fujii ${ }^{1}$, Shinji Matsumoto ${ }^{2}$, Yudai Tajiri ${ }^{1,3}$, Akira Kikuchi ${ }^{2}$ \\ and Tamotsu Kiyoshima ${ }^{1}$
}

${ }^{1}$ Laboratory of Oral Pathology, Division of Maxillofacial Diagnostic and Surgical Sciences, Faculty of Dental Science, Kyushu University, 3-1-1 Maidashi, Higashi-ku, Fukuoka 812-8582, Japan

${ }^{2}$ Department of Molecular Biology and Biochemistry, Graduate School of Medicine, Osaka University, 2-2 Yamadaoka, Suita 565-0871, Japan

${ }^{3}$ Department of Dentistry and Oral Surgery, Clinical Research Institute, National Hospital Organization Kyushu Medical Center, 1-8-1 Jigyohama, Chuo-ku, Fukuoka 810-8563, Japan

*Corresponding author. Laboratory of Oral Pathology, Division of Maxillofacial Diagnostic and Surgical Sciences, Faculty of Dental Science, Kyushu University 3-1-1 Maidashi, Higashi-ku, Fukuoka 812-8582, Japan

Phone: +81-92-642-6328; Fax: +81-92-642-6329

E-mail: sfujii@dent.kyushu-u.ac.jp 


\section{Supplementary Materials and methods}

\section{Cell lines and reagents}

Anti-PIEZO1 polyclonal antibody (15939-1-AP) (for western blotting and immunohistochemistry) was obtained from Proteintech (Rosemont, IL, USA). AntiYAP/TAZ (8418S) (for western blotting and immunocytochemistry), anti-phosphoERK1/2 (4370S), anti-ERK1/2 (4695S), anti-phospho-AKT (4060S), anti-pan-AKT (4691S) (for western blotting) and anti-cleaved caspase-3 (9664S) (for immunocytochemistry) antibodies were obtained from Cell Signaling Technology (Beverly, MA, USA). Anti-Ki-67 (ab15580) and anti-PCNA (ab92552) (for immunocytochemistry and immunohistochemistry) antibodies were from Abcam (Cambridge, UK). Anti-YAP (sc-101199) (for immunohistochemistry) antibody was from Santa Cruz Biotechnology (Dallas, TX, USA). Anti-phospho-p38 (612280) and anti-p38 (612168) (for western blotting) antibodies were from BD Biosciences (San Jose, CA, USA). Anti- $\beta$-actin (A5441) (for western blotting) antibody was from Sigma-Aldrich (Steinheim, Germany). Type I collagen solution was purchased from Nippi, Inc. (Tokyo, Japan). Phalloidin, and Simvastatin and Yoda1 were from Invitrogen and Sigma-Aldrich, respectively. GsMTx-4 was from Abcam.

\section{Plasmid construction and infection using lentivirus harboring a cDNA or ShRNA}

To generate OSCC cells that stably express $\mathrm{YAP}^{5 \mathrm{SA}}$ or PIEZO1 shRNA, parental cells (5 $\times 10^{4}$ cells/well in a 12 -well plate) were treated with lentivirus and $10 \mu \mathrm{g} / \mathrm{ml}$ polybrene. 
The cells were then centrifuged at $1080 \times \mathrm{g}$ for $1 \mathrm{~h}$, and incubated for another $24 \mathrm{~h}$. The cells that demonstrated stable expression of YAP ${ }^{5 \mathrm{SA}}$, PIEZO1 \#1 or \#2 shRNA, were selected and maintained in culture medium containing $5 \mu \mathrm{g} / \mathrm{ml}$ Blasticidin S (FUJIFILM Wako Pure Chemical Corporation, Osaka, Japan) [1].

\section{Chromatin immunoprecipitation (ChIP) assay}

ChIP assay was performed as described previously [2]. HSC-4 cells expressing Mock or $\mathrm{YAP}^{5 \mathrm{SA}}$ were cross-linked with $1 \%(\mathrm{v} / \mathrm{v})$ formaldehyde for $20 \mathrm{~min}$ at room temperature. The cells were lysed with sodium dodecyl sulfate (SDS) lysis buffer (50 mM Tris-HCl (pH 8.0), $10 \mathrm{mM}$ EDTA, and 0.5\% (w/v) SDS) and sonicated to shear DNA to a size range between 200 and $1000 \mathrm{bp}$. Sheared chromatin samples were diluted in ChIP dilution buffer (16.7 mM Tris-HCl (pH 8.0), $167 \mathrm{mM} \mathrm{NaCl}, 1.2 \mathrm{mM}$ EDTA, and 1.1\% (w/v) Triton X-100) supplemented with protease inhibitors, and precleared with salmon sperm DNA/protein A-agarose (Millipore) and incubated for $4 \mathrm{~h}$ at $4^{\circ} \mathrm{C}$ with $10 \mu \mathrm{g}$ of antiTEAD4 (ab58310, Abcam), anti-FLAG (F1804, Sigma) or negative control IgG (5415S, Cell Signaling Technology). Immunocomplexes were absorbed with salmon sperm DNA/protein A-agarose beads, and washed once with high salt buffer (20 mM Tris-HCl (pH 8.1), $500 \mathrm{mM} \mathrm{NaCl}, 0.1 \%$ (w/v) SDS, 1\% (w/v) TritonX-100, and $2 \mathrm{mM}$ EDTA), once with $\mathrm{LiCl}$ buffer (10 mM Tris-HCl (pH 8.1), 0.25 M LiCl, 1 mM EDTA, 1\% (w/v) deoxycholic acid, and 1\%(w/v) Nonidet P-40), and three times with TE buffer (10 mM Tris- $\mathrm{HCl}$ ( $\mathrm{pH} 8.1$ ), and $1 \mathrm{mM}$ EDTA). Immune complexes extracted in elution buffer (1\% (w/v) SDS and $100 \mathrm{mM} \mathrm{NaHCO}_{3}$ ) were incubated for $4 \mathrm{~h}$ at $65^{\circ} \mathrm{C}$ to revert DNA-protein 
cross-links. Then the DNA was extracted by incubation in proteinase $\mathrm{K}$ (final concentration of $50 \mathrm{mg} / \mathrm{ml}$ ) buffer for $1 \mathrm{~h}$ at $45^{\circ} \mathrm{C}$. The purified DNA was used in PCR to assess the presence of target sequences. Forward and reverse primers were as follows: fragment containing TEAD4-binding site $1, \quad 5$ 'GCGCTCATGCTTCAGGAATGC-3' and 5'-CTGCTGGCTGGTGAGTGG-3; TEAD4binding site 2, 5'-TGCCTGGAAGACTGTGTCAG-3' and 5'AATGAGACCCAGGAGGAAGGAG-3; TEAD4-binding site 3, 5'TAGGTGCTCCCTGACACTCTCTG-3' and 5'-CCAAAGCCTTCATTTCAGGA-3; ANKRD1-binding site, 5'-GAGGGGAGGACAAGCTAACC-3' and 5'CGATGTGATCACCACCAAAG-3 [3].

\section{Intracellular calcium measurement}

To examine agonist-dependent $\mathrm{Ca}^{2+}$ influx, cells were loaded with Calcium Kit-Fluo-4 $\mathrm{AM}$ (FUJIFILM Wako) for $1 \mathrm{~h}$ at $37^{\circ} \mathrm{C}$ according to the manufacturer's instructions [4]. The measurements were performed with FlexStation 3 microplate reader using the 'Flex' read mode, and images at $485 \mathrm{~nm}$ excitation/525 nm emission were collected every $2 \mathrm{sec}$. After the cells were stimulated by compound, fluorescence measurements were carried out for $5 \mathrm{~min}$. And then, we confirmed the vitality of the cells, in which propidium iodide (PI) (Wako) was not stained (data not shown).

\section{Immunocytochemistry}


HSC-4 cells were fixed in 4\% paraformaldehyde (PFA) in PBS for $30 \mathrm{~min}$ at room temperature. After cells were permeabilized in PBS containing $0.5 \%(\mathrm{w} / \mathrm{v})$ Triton X-100 and $40 \mathrm{mg} / \mathrm{ml} \mathrm{BSA} \mathrm{(Wako)} \mathrm{for} 30 \mathrm{~min}$ at room temperature, and then blocked with $0.2 \%$ BSA to prevent non-specific binding for $30 \mathrm{~min}$ at room temperature. The cells were incubated with primary antibodies (used at 1:100 for Ki-67, used at 1:300 for PCNA, used at 1:400 for cleaved caspase-3) at $4^{\circ} \mathrm{C}$ overnight, and then incubated with secondary antibodies for $3 \mathrm{~h}$ at room temperature. Cell nuclei were counterstained with 4', 6diamidino-2-phenylindole dihydrochloride (DAPI) (Dojindo, Kumamoto, Japan) or Hoechst 33342 (Dojindo) for $15 \mathrm{~min}$ at room temperature [5]. The samples were viewed with Z-X800 fluorescence microscope (Keyence, Osaka, Japan).

\section{Immunohistochemistry}

Immunohistochemical staining was performed on 5 - $\mu \mathrm{m}$-thick paraffin sections. Antigen retrieval, elimination of the endogenous peroxide activity, and blocking were carried out as previously described [4]. Then the sections were reacted with each primary antibody (used at 1:250 for PIEZO1, used at 1:100 for YAP, used at 1:300 for Ki-67, used at 1:500 for PCNA) at $4^{\circ} \mathrm{C}$ overnight. The sections were incubated with secondary antibody (Histofine Simple Stain MAX PO, Nichirei, Tokyo, Japan) for $1 \mathrm{~h}$ at RT. The immunoreactivity was visualized with a solution of DAB substrate solution. Subsequently, the sections were counterstained with hematoxylin.

\section{Microarray analysis}


Microarray analyses were performed using HSC-4 cells cultured in 2D culture or suspension culture as previously described [5]. $1 \mu \mathrm{g}$ of total RNA was used for microarray analysis. The mRNA expression profile was produced by Cell Innovator Inc. (Fukuoka, Japan) using gene microarray technology (SurePrint G3 Human Gene Expression Microarray $8 \times 60 \mathrm{~K}$ v3, Agilent Technologies, Santa Clara, CA, USA). The raw data reported in this study was deposited in NCBI GEO under accession number (GSE150044). Gene Ontology (GO) analysis was performed using DAVID database (http://david.abcc.ncifcrf.gov/). 


\section{SUPPLEMENTAL REFERENCES}

1. Matsumoto S, Fujii S, Sato A, et al. A combination of Wnt and growth factor signaling induces Arl4c expression to form epithelial tubular structures. EMBO J 2014; 33: 702-718.

2. Matsumoto S, Kurimoto T, Taketo MM, et al. The WNT/MYB pathway suppresses KIT expression to control the timing of salivary proacinar differentiation and duct formation. Development 2016; 143: 2311-2324.

3. Stein C, Bardet AF, Roma G, et al. YAP1 Exerts Its Transcriptional Control via TEAD-Mediated Activation of Enhancers. PLoS Genet 2015; 11: e1005465.

4. Fujii S, Tajiri Y, Hasegawa K, et al. The TRPV4-AKT axis promotes oral squamous cell carcinoma cell proliferation via CaMKII activation. Lab Invest 2020; 100: 311-323.

5. Fujii S, Nagata K, Matsumoto $\mathrm{S}$, et al. Wnt/ $\beta$-catenin signaling, which is activated in odontomas, reduces Sema3A expression to regulate odontogenic epithelial cell proliferation and tooth germ development. Sci Rep 2019; 9: 4257. 


\section{Supplementary Figure Legends}

Supplementary Figure 1. The effect of YAP/TAZ signaling on OSCC cells.

(A) HSC-4 cells were transfected with control or two different YAP/TAZ siRNAs for 48 h. Cell lysates were probed with anti-YAP/TAZ and anti- $\beta$-actin antibodies (upper left panel). Molecular weight size was noted referring to molecular weight marker. ANKRD1 and CYR61 mRNA levels in the cells were also measured by quantitative RT-PCR. Relative levels of ANKRD1 and CYR61 mRNA expression were normalized to GAPDH and expressed as fold-changes compared with levels in control siRNA transfected cells (upper middle panels). The cells were cultured in the presence of 5\% FBS for $48 \mathrm{~h}$, and relative cell numbers were quantified using the CyQUANT cell proliferation assay (upper right panel). RFU: relative fluorescence units. HSC-4 cells were cultured without or with 1 and $2 \mu \mathrm{M}$ Simvastatin. The cells were cultured in the presence of $5 \%$ FBS for the indicated numbers of days, and cell numbers were counted (lower left panel). ANKRD1 and CYR61 mRNA levels in the cells were also measured by quantitative RT-PCR. Relative levels of $A N K R D 1$ and CYR61 mRNA expression were normalized to GAPDH and expressed as fold-changes compared with levels in control siRNA transfected cells (lower right panel). (B) ANKRD1 and CYR61 mRNA levels in HSC-3 and MISK81-5 cells cultured in $2 \mathrm{D}$ or suspension culture for $24 \mathrm{~h}$ were measured by quantitative RTPCR. Relative levels of $A N K R D 1$ and CYR61 mRNA expression were normalized to GAPDH and expressed as fold-changes compared with expression in 2D cells. (C) HSC4 cells were transfected with control or LATS2 siRNA for $48 \mathrm{~h}$, and LATS2 mRNA levels were measured by quantitative RT-PCR. Relative LATS2 mRNA levels were normalized 
by $G A P D H$ and expressed as fold-changes compared with levels in control siRNA transfected cells. (D) HSC-4 cells were transfected with control or LATS2 siRNA for 48 h, and then cells were cultured in $2 \mathrm{D}$ or suspension culture for $24 \mathrm{~h}$. AURKB and FOXMI mRNA levels were measured by quantitative RT-PCR. Relative levels of $A U R K B$ and FOXM1 mRNA expression were normalized to $G A P D H$ and expressed as fold-changes compared with levels in 2D control cells. Results are shown as means \pm s.d. of three independent experiments. ${ }^{*} P<0.05,{ }^{*} * P<0.01$. n.s.: not significant.

Supplementary Figure 2. YAP signaling regulates PIEZO1 expression in OSCC cells. (A) The expression of PIEZO1, PIEZO2, AREG or CDK6 in 518 tumor lesions and 44 non-tumor regions in head and neck region was evaluated using TCGA dataset. Correlation between PIEZOI and AREG or CDK6 mRNA was examined in the tumor lesion. RPKM, reads per kilobase per million reads. Spearman's rank rho was used to calculate the " $\mathrm{r}$ " correlation coefficient. (B) PIEZO1 mRNA levels in HSC-3 and MISK81-5 cells cultured in 2D or suspension culture for $24 \mathrm{~h}$ were measured by quantitative RT-PCR. Relative levels of PIEZO1 mRNA expression were normalized to GAPDH and expressed as fold-changes compared with expression in 2D cells. (C) HSC3 cells were transfected with control or two different YAP/TAZ siRNAs for $48 \mathrm{~h}$, and then cells were cultured in 2D culture for $3 \mathrm{~h}$. PIEZO1 mRNA levels were measured by quantitative RT-PCR. Relative PIEZO1 mRNA levels were normalized by GAPDH and expressed as fold-changes compared with levels in control siRNA transfected cells (left panel). HSC-3 cells expressing mock or YAP ${ }^{5 \mathrm{SA}}$ were cultured in $2 \mathrm{D}$ or suspension culture 
for $24 \mathrm{~h}$ and PIEZO1 mRNA levels were measured by quantitative RT-PCR. Relative levels of PIEZO1 mRNA expression were normalized to GAPDH and expressed as foldchanges compared with expression in 2D control cells (right panel). (D) HSC-4 cells expressing mock or $\mathrm{YAP}^{5 \mathrm{SA}}$ were cultured in $2 \mathrm{D}$ or suspension culture for $24 \mathrm{~h}$, and ANKRD1 and CYR61 mRNA levels were measured by quantitative RT-PCR. Relative levels of ANKRD1 and CYR61 mRNA expression were normalized to GAPDH and expressed as fold-changes compared with expression in 2D control cells. (E) Genomic views of TEAD1, TEAD4, H3K27ac and H3K9ac ChIP enrichment at human PIEZO1 gene obtained from Cistrome Data Browser database. The PIEZOI gene enhancer region contains three predicted TEAD4-binding sites. Chromatin from HSC-4 cells expressing mock was immunoprecipitated with anti-control IgG or anti-TEAD4 antibody, and the precipitates were analyzed by PCR for TEAD4 binding site of the ANKRD1 gene promoter. Results are shown as means \pm s.d. of three independent experiments. ${ }^{*} P<0.05$, $* * P<0.01$. n.s.: not significant.

Supplementary Figure 3. The effect of PIEZO1 on YAP/TAZ signaling.

(A) The expression of PIEZO1 and PIEZO2 in cell lines was evaluated using Cancer Cell Line Encyclopedia dataset. (B) PIEZO2 mRNA levels were measured in OSCC cell lines (HSC-4, HSC-3, HSC-2, SAS, MISK81-5 and sMISK cells), A549 and MKN-45 cells by quantitative RT-PCR. Relative levels of PIEZO2 mRNA expression were normalized to GAPDH and expressed as fold-changes compared with expression in control A549 cells. (C) HSC-4 cells were pre-loaded with Fluo-4-AM and were exposed without or with 1 
and $10 \mu \mathrm{M}$ Yoda1. Then, intracellular $\mathrm{Ca}^{2+}$ influx was measured. RFU: relative fluorescence units. (D) HSC-4 cells were transfected with control or two different PIEZO1 siRNAs for $48 \mathrm{~h}$, and PIEZO2, ANKRD1 and CYR61 mRNA levels were measured by quantitative RT-PCR. Relative PIEZO2, ANKRD1 and CYR61 mRNA levels were normalized by GAPDH and expressed as fold-changes compared with levels in control siRNA transfected cells (upper panels). HSC-4 cells were transfected with control or two different PIEZO1 siRNAs for $48 \mathrm{~h}$, and the cells were stained with anti-YAP/TAZ antibody, phalloidin and Hoechst 33342. Cells with nuclear YAP/TAZ are expressed as the percentage of YAP/TAZ-positively stained cells compared with total Hoechst 33342stained cells $(n=1,516)$ (lower panels). (E) HSC-4 cells transfected with control or two different PIEZO1 siRNAs were pre-loaded with Fluo-4-AM and were exposed without or with $10 \mu \mathrm{M}$ Yoda1. Then, intracellular $\mathrm{Ca}^{2+}$ influx was measured. RFU: relative fluorescence units. Results are shown as means \pm s.d. of three independent experiments. Scale bars, $100 \mu \mathrm{m}$. n.s.: not significant.

Supplementary Figure 4. PIEZO1 regulates cell proliferation of OSCC cells.

(A) HSC-4 cells were transfected with control or PIEZO1 \#1 siRNA for $48 \mathrm{~h}$. The cells were stained with anti-PCNA antibody and Hoechst 33342, and then PCNA-positive cells and Hoechst 33342-stained cells were counted, respectively. Results are expressed as the percentage of PCNA-positive cells compared with total Hoechst 33342-stained cells. (B) HSC-4 cells were transfected with control or PIEZO1 \#1 siRNA for $48 \mathrm{~h}$. The cells were stained with anti-cleaved caspase-3 antibody and DAPI. (C) HSC-4 cells expressing 
control or PIEZO1 \#1 and \#2 shRNAs were pre-loaded with Fluo-4-AM and were exposed without or with $10 \mu \mathrm{M}$ Yoda1. Then, intracellular $\mathrm{Ca}^{2+}$ influx was measured. RFU: relative fluorescence units. (D) HSC-4 cells expressing control or PIEZO1 \#1 and \#2 shRNAs were transfected with control or LATS2 siRNA for $48 \mathrm{~h}$ (left panels) or with mock or YAP ${ }^{5 \mathrm{SA}}$ expression (right panels), and then cells were cultured for 4 days in the 3D Matrigel. The cells were stained with anti-PCNA antibody and Hoechst 33342, and then PCNA-positive cells and Hoechst 33342-stained cells were counted, respectively. Results are expressed as the percentage of PCNA-positively stained cells compared with total Hoechst 33342-stained cells $(n=14,242)$. (E) HSC-4 cells expressing PIEZO1 \#1 and \#2 shRNAs were transfected with control or LATS2 siRNA for $48 \mathrm{~h}$ (left panels) or with mock or $\mathrm{YAP}^{5 \mathrm{SA}}$ expression (right panels), and then cells were cultured in $2 \mathrm{D}$ or suspension culture for $24 \mathrm{~h}$. PIEZOI or ANKRD1 mRNA levels were measured by quantitative RT-PCR. Relative PIEZO1 or ANKRD1 mRNA levels were normalized by $G A P D H$ and expressed as fold-changes compared with levels in 2D control cells. Results are shown as means \pm s.d. of three independent experiments. ${ }^{*} P<0.05,{ }^{*} P<0.01$. Scale bars, $100 \mu \mathrm{m}$. n.s.: not significant.

Supplementary Figure 5. PIEZO1 is expressed in human oral squamous cell carcinoma tissues.

Oral squamous cell carcinoma tissues $(n=45)$ were stained with anti-PIEZO1 antibody and hematoxylin. PIEZO1-positive signal intensity was classified as follows: negative; weak; strong. Boxes show enlarged images. Scale bars, $100 \mu \mathrm{m}$. 
Supplementary Figure 6. YAP is expressed in human oral squamous cell carcinoma tissues.

(A) Oral squamous cell carcinoma tissues $(n=45)$ were stained with anti-YAP antibody and hematoxylin. YAP expression and localization were classified as follows: negative; nucleus/cytoplasm; nucleus. Boxes show enlarged images. (B) Percentages of three groups based on the YAP expression and localization in the tumor lesions and the nontumor regions are shown in the panel. (C) Oral squamous cell carcinoma tissues $(n=45)$ were stained with anti-YAP, anti-PIEZO1 and anti-Ki-67 antibodies, and hematoxylin. Box shows enlarged images. Scale bars, $50 \mu \mathrm{m}$.

Supplementary Figure 7. Co-expression of PIEZO1, YAP, Ki-67 and PCNA in human oral squamous cell carcinoma tissues.

Oral squamous cell carcinoma tissues $(n=45)$ were stained with anti-PIEZO1, anti- YAP, anti-Ki-67 and anti-PCNA antibodies, and hematoxylin. Representative data $(n=5)$ are shown. Scale bars, $20 \mu \mathrm{m}$. 
Table S1. Designs for siRNAs used in this study.

\begin{tabular}{|c|c|}
\hline siRNA & Sequence \\
\hline human YAP \#1 & $\begin{array}{l}\text { Sense: GACAUCUUCUGGUCAGAGATT } \\
\text { Antisense: UCUCUGACCAGAAGAUGUCTT }\end{array}$ \\
\hline human YAP \#2 & $\begin{array}{l}\text { Sense: GCUCAUUCCUCUCCAGCUUTT } \\
\text { Antisense: AAGCUGGAGAGGAAUGAGCTT }\end{array}$ \\
\hline human TAZ \#1 & $\begin{array}{l}\text { Sense: AGGUACUUCCUCAAUCACATT } \\
\text { Antisense: UGUGAUUGAGGAAGUACCUTT }\end{array}$ \\
\hline human TAZ \#2 & $\begin{array}{l}\text { Sense: CCCAACAGACCCGUUUCCCUGAUUUTT } \\
\text { Antisense: AAAUCAGGGAAACGGGUCUGUUGGGTT }\end{array}$ \\
\hline human LATS2 & $\begin{array}{l}\text { Sense: GCCACGACUUAUUCUGGAATT } \\
\text { Antisense: UUCCAGAAUAAGUCGUGGCTT }\end{array}$ \\
\hline human PIEZO1 \#1 & $\begin{array}{l}\text { Sense: AGAAGAAGAUCGUCAAGUATT } \\
\text { Antisense: UACUUGACGAUCUUCUUCUTT }\end{array}$ \\
\hline human PIEZO1 \#2 & $\begin{array}{l}\text { Sense: CCAACCUCAUCAGCGACUUTT } \\
\text { Antisense: AAGUCGCUGAUGAGGUUGGTT }\end{array}$ \\
\hline $\begin{array}{l}\text { Randomized } \\
\text { control }\end{array}$ & $\begin{array}{l}\text { Sense: CAGUCGCGUUUGCGACUGGTT } \\
\text { Antisense: CCAGUCGCAAACGCGACUGTT }\end{array}$ \\
\hline
\end{tabular}


Table S2. Quantitative RT-PCR primers used in this study.

\begin{tabular}{|l|l|}
\hline Gene & Primers \\
\hline human LATS2 & $\begin{array}{l}\text { Forward 5'-GCGATTCGTTTGCGTCCTAC-3' } \\
\text { Reverse 5'-CCGGGGAAAAGGTAGTCCAC-3' }\end{array}$ \\
\hline human PIEZO1 & $\begin{array}{l}\text { Forward 5'-AGGAACAGGCAGGACAGCTA-3' } \\
\text { Reverse 5'-GCGTGTCAGCTCATCCACTA-3' }\end{array}$ \\
\hline human PIEZO2 & $\begin{array}{l}\text { Forward 5'-GACGACTCTGCAGGCAAGAA-3' } \\
\text { Reverse 5'-AGAAGAGCAGCAGAAATCGGG-3' }\end{array}$ \\
\hline human ANKRD1 & $\begin{array}{l}\text { Forward 5'-ACGCCAAAGACAGAGAAGGA-3' } \\
\text { Reverse 5'-TTCTGCCAGTGTAGCACCAG-3' }\end{array}$ \\
\hline human CYR61 & $\begin{array}{l}\text { Forward 5'-TCCGAGGTGGAGTTGACGAG-3' } \\
\text { Reverse 5'-AGCACTGGGACCATGAAGTTG-3' }\end{array}$ \\
\hline human AURKB & $\begin{array}{l}\text { Forward 5'-CCTCCCTGAGGAGGAAGACA-3' } \\
\text { Reverse 5'-TGCACCACAGATCCACCT TC-3' }\end{array}$ \\
\hline human FOXM1 & $\begin{array}{l}\text { Forward 5'-GTTTATCAGTGCTGCTAGCTGAGG-3' } \\
\text { Reverse 5'-CAGGCTGGATTTCTTCCTCCTTG-3' }\end{array}$ \\
\hline human GAPDH & $\begin{array}{l}\text { Forward 5'-GCACCGTCAAGGCTGAGAAC-3' } \\
\text { Reverse 5'-TGGTGAAGACGCCAGTGGA-3' }\end{array}$ \\
\hline
\end{tabular}


Table S3. Gene enrichment analysis in HSC-4 cells.

\begin{tabular}{|c|c|c|c|}
\hline Term & Count & $\%$ & $p$-Value \\
\hline DNA replication & 40 & 3.487358 & $1.53 \mathrm{E}-16$ \\
\hline G1/S transition of mitotic cell cycle & 32 & 2.789887 & 7.64E-16 \\
\hline rRNA processing & 46 & 4.010462 & $1.28 \mathrm{E}-15$ \\
\hline Mitotic nuclear division & 49 & 4.272014 & 4.49E-15 \\
\hline Cell division & 59 & 5.143854 & 8.91E-15 \\
\hline Sister chromatid cohesion & 30 & 2.615519 & $5.89 \mathrm{E}-14$ \\
\hline DNA replication initiation & 17 & 1.482127 & $1.00 \mathrm{E}-12$ \\
\hline $\begin{array}{l}\text { Regulation of transcription involved in G1/S transition of } \\
\text { mitotic cell cycle }\end{array}$ & 11 & 0.959024 & 1.07E-07 \\
\hline Regulation of cell cycle & 24 & 2.092415 & $1.42 \mathrm{E}-07$ \\
\hline Cell proliferation & 46 & 4.010462 & 1.46E-07 \\
\hline Chromosome segregation & 17 & 1.482127 & 3.99E-07 \\
\hline De novo' IMP biosynthetic process & 6 & 0.523104 & 2.44E-06 \\
\hline DNA unwinding involved in DNA replication & 7 & 0.610288 & 3.94E-06 \\
\hline Purine nucleotide biosynthetic process & 7 & 0.610288 & $1.58 \mathrm{E}-05$ \\
\hline $\begin{array}{l}\text { Purine ribonucleoside monophosphate biosynthetic } \\
\text { process }\end{array}$ & 7 & 0.610288 & 2.80E-05 \\
\hline Mitotic sister chromatid segregation & 9 & 0.784656 & 3.03E-05 \\
\hline Telomere maintenance via recombination & 10 & 0.87184 & 3.08E-05 \\
\hline Response to drug & 35 & 3.051439 & 3.63E-05 \\
\hline Response to unfolded protein & 11 & 0.959024 & 5.44E-05 \\
\hline DNA repair & 29 & 2.528335 & 5.73E-05 \\
\hline DNA synthesis involved in DNA repair & 10 & 0.87184 & $6.71 \mathrm{E}-05$ \\
\hline DNA strand elongation involved in DNA replication & 7 & 0.610288 & 7.45E-05 \\
\hline Pyrimidine nucleoside biosynthetic process & 5 & 0.43592 & 1.10E-04 \\
\hline Spindle organization & 7 & 0.610288 & 1.14E-04 \\
\hline Organ regeneration & 11 & 0.959024 & $1.50 \mathrm{E}-04$ \\
\hline Cellular response to hypoxia & 16 & 1.394943 & $1.62 \mathrm{E}-04$ \\
\hline rRNA transcription & 6 & 0.523104 & $2.46 \mathrm{E}-04$ \\
\hline Sprouting angiogenesis & 8 & 0.697472 & $2.46 \mathrm{E}-04$ \\
\hline Ribosomal large subunit biogenesis & 8 & 0.697472 & 2.46E-04 \\
\hline Strand displacement & 8 & 0.697472 & $3.21 \mathrm{E}-04$ \\
\hline Canonical glycolysis & 8 & 0.697472 & 3.21E-04 \\
\hline Response to hypoxia & 22 & 1.918047 & 3.34E-04 \\
\hline G2/M transition of mitotic cell cycle & 19 & 1.656495 & 3.56E-04 \\
\hline Positive regulation of gene expression, epigenetic & 12 & 1.046207 & 3.87E-04 \\
\hline Response to starvation & 9 & 0.784656 & 4.10E-04 \\
\hline DNA replication checkpoint & 5 & 0.43592 & $4.72 \mathrm{E}-04$ \\
\hline
\end{tabular}




\begin{tabular}{|c|c|c|c|}
\hline $\begin{array}{l}\text { Double-strand break repair via homologous } \\
\text { recombination }\end{array}$ & 13 & 1.133391 & $5.05 \mathrm{E}-04$ \\
\hline Mitotic metaphase plate congression & 9 & 0.784656 & 6.12E-04 \\
\hline $\begin{array}{l}\text { Positive regulation of establishment of protein } \\
\text { localization to telomere }\end{array}$ & 5 & 0.43592 & 8.14E-04 \\
\hline $\begin{array}{l}\text { Positive regulation of telomerase RNA localization to } \\
\text { Cajal body }\end{array}$ & 6 & 0.523104 & 8.16E-04 \\
\hline Maturation of LSU-rRNA & 6 & 0.523104 & 8.16E-04 \\
\hline Mitotic spindle organization & 8 & 0.697472 & 8.26E-04 \\
\hline Regulation of signal transduction by p53 class mediator & 17 & 1.482127 & 9.05E-04 \\
\hline $\begin{array}{l}\text { Maturation of SSU-rRNA from tricistronic rRNA } \\
\text { transcript (SSU-rRNA, 5.8S rRNA, LSU-rRNA) }\end{array}$ & 8 & 0.697472 & 0.001244 \\
\hline $\begin{array}{l}\text { Positive regulation of telomere maintenance via } \\
\text { telomerase }\end{array}$ & 8 & 0.697472 & 0.001244 \\
\hline Chaperone mediated protein folding requiring cofactor & 5 & 0.43592 & 0.0013 \\
\hline Purine nucleobase biosynthetic process & 4 & 0.348736 & 0.001392 \\
\hline Cellular response to DNA damage stimulus & 23 & 2.005231 & 0.001706 \\
\hline CENP-A containing nucleosome assembly & 9 & 0.784656 & 0.001733 \\
\hline DNA duplex unwinding & 9 & 0.784656 & 0.002021 \\
\hline Double-strand break repair & 11 & 0.959024 & 0.002456 \\
\hline Toxin transport & 8 & 0.697472 & 0.002563 \\
\hline Ribosome biogenesis & 8 & 0.697472 & 0.002563 \\
\hline Cell cycle & 23 & 2.005231 & 0.0029 \\
\hline Spliceosomal snRNP assembly & 7 & 0.610288 & 0.003093 \\
\hline Cytokinesis & 9 & 0.784656 & 0.003567 \\
\hline Mitotic cytokinesis & 7 & 0.610288 & 0.003727 \\
\hline Protein sumoylation & 15 & 1.307759 & 0.003749 \\
\hline Protein folding in endoplasmic reticulum & 5 & 0.43592 & 0.003892 \\
\hline $\begin{array}{l}\text { Regulation of cyclin-dependent protein serine/threonine } \\
\text { kinase activity }\end{array}$ & 8 & 0.697472 & 0.00411 \\
\hline Cellular response to gamma radiation & 6 & 0.523104 & 0.004234 \\
\hline Positive regulation of cell migration & 20 & 1.743679 & 0.004437 \\
\hline Response to testosterone & 7 & 0.610288 & 0.004452 \\
\hline DNA damage checkpoint & 7 & 0.610288 & 0.004452 \\
\hline $\begin{array}{l}\text { Positive regulation of mitotic cell cycle spindle assembly } \\
\text { checkpoint }\end{array}$ & 4 & 0.348736 & 0.004493 \\
\hline Maturation of SSU-rRNA & 5 & 0.43592 & 0.005221 \\
\hline Mitotic cell cycle checkpoint & 7 & 0.610288 & 0.006207 \\
\hline Lactation & 8 & 0.697472 & 0.006279 \\
\hline Protein heterotetramerization & 8 & 0.697472 & 0.006279 \\
\hline Regulation of chromosome segregation & 4 & 0.348736 & 0.006905 \\
\hline Positive regulation of protein localization to Cajal body & 4 & 0.348736 & 0.006905 \\
\hline
\end{tabular}




\begin{tabular}{|c|c|c|c|}
\hline Mitotic spindle midzone assembly & 4 & 0.348736 & 0.006905 \\
\hline $\begin{array}{l}\text { Endonucleolytic cleavage in ITS1 to separate SSU- } \\
\text { rRNA from 5.8S rRNA and LSU-rRNA from tricistronic } \\
\text { rRNA transcript (SSU-rRNA, 5.8S rRNA, LSU-rRNA) }\end{array}$ & 4 & 0.348736 & 0.006905 \\
\hline Negative regulation of B cell apoptotic process & 4 & 0.348736 & 0.006905 \\
\hline Regulation of dendrite development & 4 & 0.348736 & 0.006905 \\
\hline Cellular response to UV & 8 & 0.697472 & 0.008136 \\
\hline Mitotic nuclear envelope disassembly & 8 & 0.697472 & 0.008136 \\
\hline Histone H3-K9 dimethylation & 3 & 0.261552 & 0.008212 \\
\hline De novo' UMP biosynthetic process & 3 & 0.261552 & 0.008212 \\
\hline Epithelial to mesenchymal transition & 7 & 0.610288 & 0.008417 \\
\hline Ribosomal small subunit biogenesis & 5 & 0.43592 & 0.008719 \\
\hline Cerebral cortex development & 9 & 0.784656 & 0.009245 \\
\hline Base-excision repair & 7 & 0.610288 & 0.009711 \\
\hline Cholesterol metabolic process & 10 & 0.87184 & 0.00975 \\
\hline Attachment of spindle microtubules to kinetochore & 4 & 0.348736 & 0.009951 \\
\hline \begin{tabular}{|l|} 
Maturation of LSU-rRNA from tricistronic rRNA \\
transcript (SSU-rRNA, 5.8S rRNA, LSU-rRNA)
\end{tabular} & 4 & 0.348736 & 0.009951 \\
\hline ATF6-mediated unfolded protein response & 4 & 0.348736 & 0.009951 \\
\hline Protein maturation by protein folding & 4 & 0.348736 & 0.009951 \\
\hline Nucleosome assembly & 14 & 1.220575 & 0.010831 \\
\hline Negative regulation of transcription, DNA-templated & 40 & 3.487358 & 0.010879 \\
\hline DNA biosynthetic process & 6 & 0.523104 & 0.010982 \\
\hline DNA recombination & 11 & 0.959024 & 0.01261 \\
\hline $\begin{array}{l}\text { Positive regulation of vascular endothelial growth factor } \\
\text { production }\end{array}$ & 6 & 0.523104 & 0.0129 \\
\hline Microtubule polymerization & 4 & 0.348736 & 0.013658 \\
\hline Microtubule depolymerization & 4 & 0.348736 & 0.013658 \\
\hline Interstrand cross-link repair & 8 & 0.697472 & 0.014507 \\
\hline Peptidyl-arginine N-methylation & 3 & 0.261552 & 0.015843 \\
\hline scaRNA localization to Cajal body & 3 & 0.261552 & 0.015843 \\
\hline Kidney development & 11 & 0.959024 & 0.015931 \\
\hline Negative regulation of DNA replication & 5 & 0.43592 & 0.016355 \\
\hline Nucleoside metabolic process & 5 & 0.43592 & 0.016355 \\
\hline $\begin{array}{l}\text { DNA damage response, signal transduction by p53 } \\
\text { class mediator resulting in cell cycle arrest }\end{array}$ & 9 & 0.784656 & 0.016662 \\
\hline Positive regulation of telomerase activity & 6 & 0.523104 & 0.017387 \\
\hline $\begin{array}{l}\text { Double-strand break repair via nonhomologous end } \\
\text { joining }\end{array}$ & 9 & 0.784656 & 0.018223 \\
\hline Mitotic spindle assembly checkpoint & 5 & 0.43592 & 0.019601 \\
\hline G2 DNA damage checkpoint & 5 & 0.43592 & 0.019601 \\
\hline Glutamine metabolic process & 5 & 0.43592 & 0.019601 \\
\hline
\end{tabular}




\begin{tabular}{|c|c|c|c|}
\hline $\begin{array}{l}\text { Intrinsic apoptotic signaling pathway in response to } \\
\text { DNA damage by p53 class mediator }\end{array}$ & 6 & 0.523104 & 0.022801 \\
\hline $\begin{array}{l}\text { Negative regulation of transcription from RNA } \\
\text { polymerase II promoter }\end{array}$ & 52 & 4.533566 & 0.023118 \\
\hline Metaphase plate congression & 4 & 0.348736 & 0.023124 \\
\hline PERK-mediated unfolded protein response & 4 & 0.348736 & 0.023124 \\
\hline Positive regulation of fibroblast proliferation & 8 & 0.697472 & 0.023821 \\
\hline Histone $\mathrm{H} 3-\mathrm{K} 9$ trimethylation & 3 & 0.261552 & 0.025478 \\
\hline AMP biosynthetic process & 3 & 0.261552 & 0.025478 \\
\hline Ribonucleoside monophosphate biosynthetic process & 3 & 0.261552 & 0.025478 \\
\hline $\begin{array}{l}\text { Ddouble-strand break repair via break-induced } \\
\text { replication }\end{array}$ & 3 & 0.261552 & 0.025478 \\
\hline Cellular response to xenobiotic stimulus & 3 & 0.261552 & 0.025478 \\
\hline Regulation of striated muscle tissue development & 3 & 0.261552 & 0.025478 \\
\hline Response to inorganic substance & 3 & 0.261552 & 0.025478 \\
\hline Response to activity & 7 & 0.610288 & 0.025493 \\
\hline DNA replication-dependent nucleosome assembly & 6 & 0.523104 & 0.025874 \\
\hline Positive regulation of neutrophil chemotaxis & 5 & 0.43592 & 0.027211 \\
\hline Positive regulation of protein complex assembly & 5 & 0.43592 & 0.027211 \\
\hline Chaperone-mediated protein complex assembly & 4 & 0.348736 & 0.028894 \\
\hline Protein stabilization & 14 & 1.220575 & 0.029622 \\
\hline Protein folding & 17 & 1.482127 & 0.031226 \\
\hline NLS-bearing protein import into nucleus & 5 & 0.43592 & 0.031591 \\
\hline Negative regulation of myoblast differentiation & 5 & 0.43592 & 0.031591 \\
\hline $\begin{array}{l}\text { Regulation of ubiquitin-protein ligase activity involved in } \\
\text { mitotic cell cycle }\end{array}$ & 5 & 0.43592 & 0.031591 \\
\hline $\begin{array}{l}\text { Transmembrane receptor protein tyrosine kinase } \\
\text { signaling pathway }\end{array}$ & 11 & 0.959024 & 0.031708 \\
\hline Epithelial cell differentiation & 9 & 0.784656 & 0.032184 \\
\hline Glycolytic process & 6 & 0.523104 & 0.032782 \\
\hline Negative regulation of smooth muscle cell migration & 4 & 0.348736 & 0.035351 \\
\hline Oxidation-reduction process & 43 & 3.74891 & 0.036266 \\
\hline Meiotic nuclear division & 5 & 0.43592 & 0.036359 \\
\hline Regulation of mitotic nuclear division & 5 & 0.43592 & 0.036359 \\
\hline Cellular response to interleukin-4 & 5 & 0.43592 & 0.036359 \\
\hline Binding of sperm to zona pellucida & 6 & 0.523104 & 0.036627 \\
\hline Tetrahydrofolate biosynthetic process & 3 & 0.261552 & 0.03688 \\
\hline DNA replication, synthesis of RNA primer & 3 & 0.261552 & 0.03688 \\
\hline $\begin{array}{l}\text { Endonucleolytic cleavage in 5'-ETS of tricistronic rRNA } \\
\text { transcript (SSU-rRNA, 5.8S rRNA, LSU-rRNA) }\end{array}$ & 3 & 0.261552 & 0.03688 \\
\hline Positive regulation of exit from mitosis & 3 & 0.261552 & 0.03688 \\
\hline De novo' pyrimidine nucleobase biosynthetic process & 3 & 0.261552 & 0.03688 \\
\hline
\end{tabular}




\begin{tabular}{|l|c|c|c|}
\hline Histone H4-R3 methylation & 3 & 0.261552 & 0.03688 \\
\hline Response to cold & 6 & 0.523104 & 0.040738 \\
\hline Mitotic spindle assembly & 6 & 0.523104 & 0.040738 \\
\hline Microtubule bundle formation & 5 & 0.43592 & 0.041519 \\
\hline Response to cadmium ion & 5 & 0.43592 & 0.041519 \\
\hline Positive regulation of phosphorylation & 5 & 0.43592 & 0.041519 \\
\hline Nucleobase-containing small molecule interconversion & 5 & 0.43592 & 0.041519 \\
\hline Cell migration & 16 & 1.394943 & 0.041694 \\
\hline Protein refolding & 4 & 0.348736 & 0.042483 \\
\hline Response to copper ion & 4 & 0.348736 & 0.042483 \\
\hline Positive regulation of angiogenesis & 12 & 1.046207 & 0.042934 \\
\hline Response to ionizing radiation & 7 & 0.610288 & 0.044815 \\
\hline Nucleobase-containing compound metabolic process & 7 & 0.610288 & 0.044815 \\
\hline Adherens junction organization & 6 & 0.523104 & 0.045117 \\
\hline Chromatin silencing at rDNA & 6 & 0.523104 & 0.045117 \\
\hline Response to endoplasmic reticulum stress & 9 & 0.784656 & 0.045757 \\
\hline Regulation of cellular response to heat & 9 & 0.784656 & 0.045757 \\
\hline tRNA methylation & 5 & 0.43592 & 0.047072 \\
\hline Positive regulation of protein tyrosine kinase activity & 5 & 0.43592 & 0.047072 \\
\hline DNA replication-independent nucleosome assembly & 5 & 0.43592 & 0.047072 \\
\hline Box C/D snoRNP assembly & 3 & 0.261552 & 0.049836 \\
\hline Axon ensheathment & 3 & 0.261552 & 0.049836 \\
\hline Strand invasion & 0.261552 & 0.049836 \\
\hline
\end{tabular}


Table S4. List of gene from microarray analysis data of HSC-4 cells cultured in 2D culture and suspension culture.

\begin{tabular}{|c|c|c|}
\hline Gene Symbol & EntrezGeneID & $\begin{array}{c}\text { Ratio } \\
\text { 2D culture vs } \\
\text { suspension culture }\end{array}$ \\
\hline AREG & 374 & 0.17 \\
\hline AURKB & 9212 & 0.04 \\
\hline CCND1 & 595 & 0.52 \\
\hline CDK6 & 1021 & 0.43 \\
\hline E2F1 & 1869 & 0.05 \\
\hline FOXM1 & 2305 & 0.10 \\
\hline KIF23 & 9493 & 0.08 \\
\hline MCM7 & 4176 & 0.07 \\
\hline PIEZO1 & 9780 & 0.94 \\
\hline PIEZO2 & 63895 & NULL \\
\hline & & \\
\hline
\end{tabular}


The Journal of Pathbldassegawa et al., Figure S1
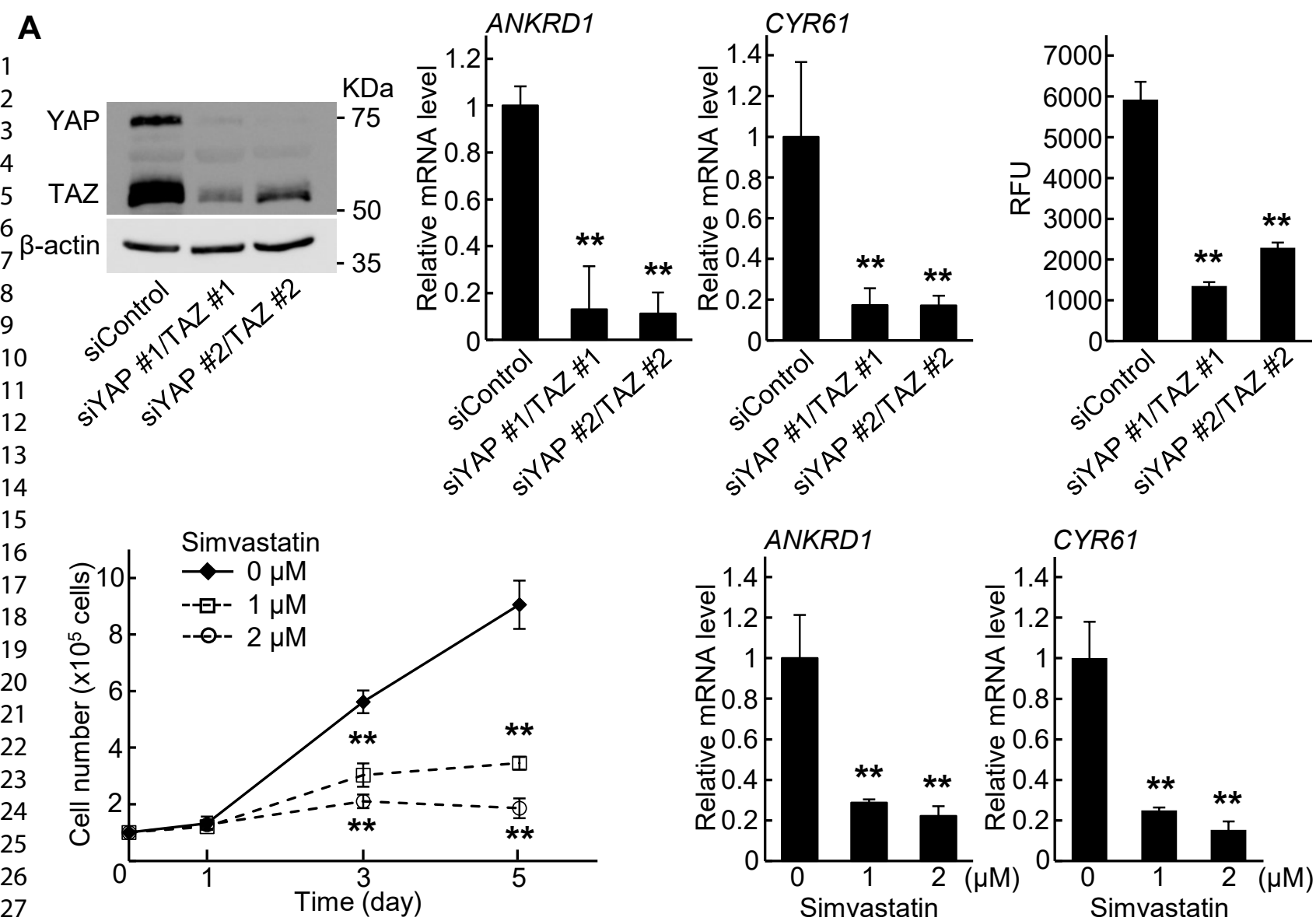

ANKRD1

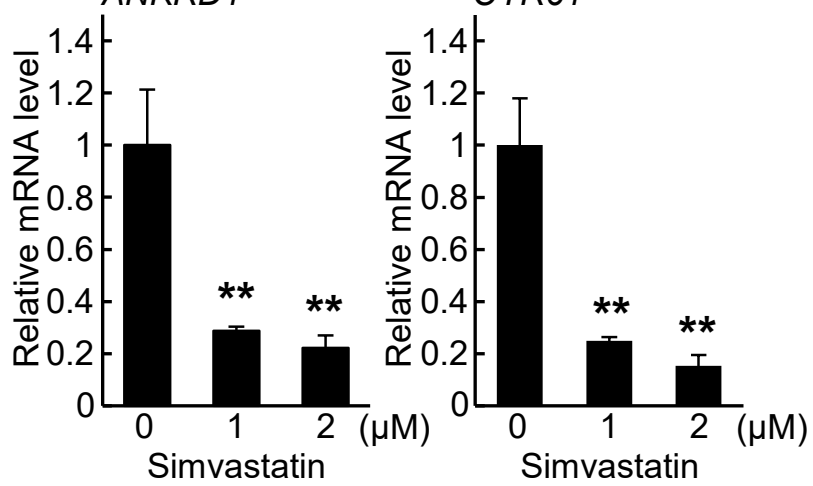

$2 \&$
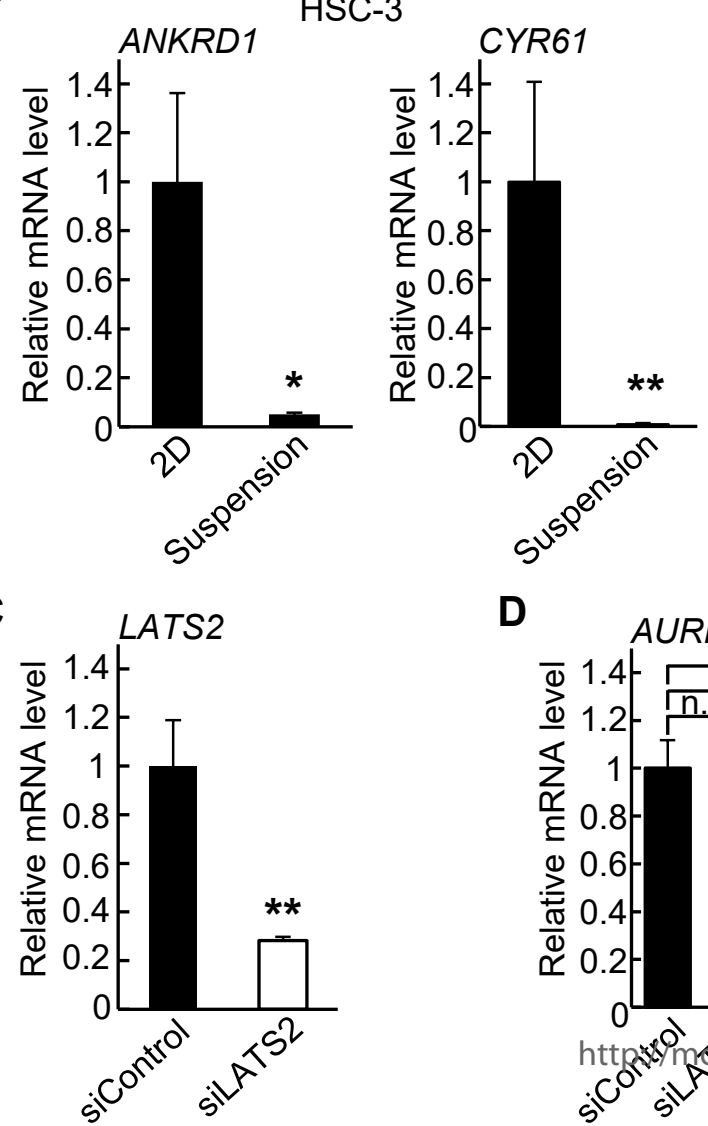

D




Supplementary Figure 1. The effect of YAP/TAZ signaling on OSCC cells.

(A) HSC-4 cells were transfected with control or two different YAP/TAZ siRNAs for $48 \mathrm{~h}$. Cell lysates were probed with anti-YAP/TAZ and anti- $\beta$-actin antibodies (upper left panel). Molecular weight size was noted referring to molecular weight marker. ANKRD1 and CYR61 mRNA levels in the cells were also measured by quantitative RT-PCR. Relative levels of ANKRD1 and CYR61 mRNA expression were normalized to GAPDH and expressed as fold-changes compared with levels in control siRNA transfected cells (upper middle panels). The cells were cultured in the presence of 5\% FBS for $48 \mathrm{~h}$, and relative cell numbers were quantified using the CyQUANT cell proliferation assay (upper right panel). RFU: relative fluorescence units. HSC-4 cells were cultured without or with 1 and $2 \mu \mathrm{M}$ Simvastatin. The cells were cultured in the presence of $5 \%$ FBS for the indicated numbers of days, and cell numbers were counted (lower left panel). ANKRD1 and CYR61 mRNA levels in the cells were also measured by quantitative RT-PCR. Relative levels of ANKRD1 and CYR61 mRNA expression were normalized to GAPDH and expressed as fold-changes compared with levels in control siRNA transfected cells (lower right panel). (B) ANKRD1 and CYR61 mRNA levels in HSC-3 and MISK81-5 cells cultured in 2D or suspension culture for $24 \mathrm{~h}$ were measured by quantitative RT-PCR. Relative levels of $A N K R D 1$ and CYR61 mRNA expression were normalized to GAPDH and expressed as fold-changes compared with expression in 2D cells. (C) HSC-4 cells were transfected with control or LATS2 siRNA for $48 \mathrm{~h}$, and LATS2 mRNA levels were measured by quantitative RT-PCR. Relative LATS2 mRNA levels were normalized by GAPDH and expressed as fold-changes compared with levels in control siRNA transfected cells. (D) HSC-4 cells were transfected with control or LATS2 siRNA for $48 \mathrm{~h}$, and then cells were cultured in 2D or suspension culture for $24 \mathrm{~h}$. $A U R K B$ and FOXM1 mRNA levels were measured by quantitative RT-PCR. Relative levels of $A U R K B$ and FOXM1 mRNA expression were normalized to GAPDH and expressed as foldchanges compared with levels in $2 \mathrm{D}$ control cells. Results are shown as means \pm s.d. of three independent experiments. ${ }^{*} P<0.05, * * P<0.01$. n.s.: not significant. 
$1 \quad$ Supplementary Figure 2. YAP signaling regulates PIEZO1 expression in OSCC cells.

(A) The expression of PIEZO1, PIEZO2, AREG or CDK6 in 518 tumor lesions and 44 nontumor regions in head and neck region was evaluated using TCGA dataset. Correlation between PIEZO1 and AREG or CDK6 mRNA was examined in the tumor lesion. RPKM, reads per kilobase per million reads. Spearman's rank rho was used to calculate the " $r$ " correlation coefficient. (B) PIEZO1 mRNA levels in HSC-3 and MISK81-5 cells cultured in 2D or suspension culture for $24 \mathrm{~h}$ were measured by quantitative RT-PCR. Relative levels of PIEZOI mRNA expression were normalized to $G A P D H$ and expressed as fold-changes compared with expression in 2D cells. (C) HSC-3 cells were transfected with control or two different YAP/TAZ siRNAs for $48 \mathrm{~h}$, and then cells were cultured in 2D culture for $3 \mathrm{~h}$. PIEZO1 mRNA levels were measured by quantitative RT-PCR. Relative PIEZO1 mRNA levels were normalized by GAPDH and expressed as fold-changes compared with levels in control siRNA transfected cells (left panel). HSC-3 cells expressing mock or YAP ${ }^{5 \mathrm{SA}}$ were cultured in 2D or suspension culture for 24 $\mathrm{h}$ and PIEZO1 mRNA levels were measured by quantitative RT-PCR. Relative levels of PIEZO1 mRNA expression were normalized to GAPDH and expressed as fold-changes compared with expression in 2D control cells (right panel). (D) HSC-4 cells expressing mock or YAP ${ }^{5 S A}$ were cultured in 2D or suspension culture for $24 \mathrm{~h}$, and $A N K R D 1$ and CYR61 mRNA levels were measured by quantitative RT-PCR. Relative levels of $A N K R D 1$ and CYR61 mRNA expression were normalized to GAPDH and expressed as fold-changes compared with expression in 2D control cells. (E) Genomic views of TEAD1, TEAD4, H3K27ac and H3K9ac ChIP enrichment at human PIEZO1 gene obtained from Cistrome Data Browser database. The PIEZO1 gene enhancer region contains three predicted TEAD4-binding sites. Chromatin from HSC-4 cells expressing mock was immunoprecipitated with anti-control IgG or anti-TEAD4 antibody, and the precipitates were analyzed by PCR for TEAD4 binding site of the ANKRD1 gene promoter. Results are shown as means \pm s.d. of three independent experiments. ${ }^{*} P<0.05, * * P<0.01$. n.s.: not significant. 
Page 69 of 77

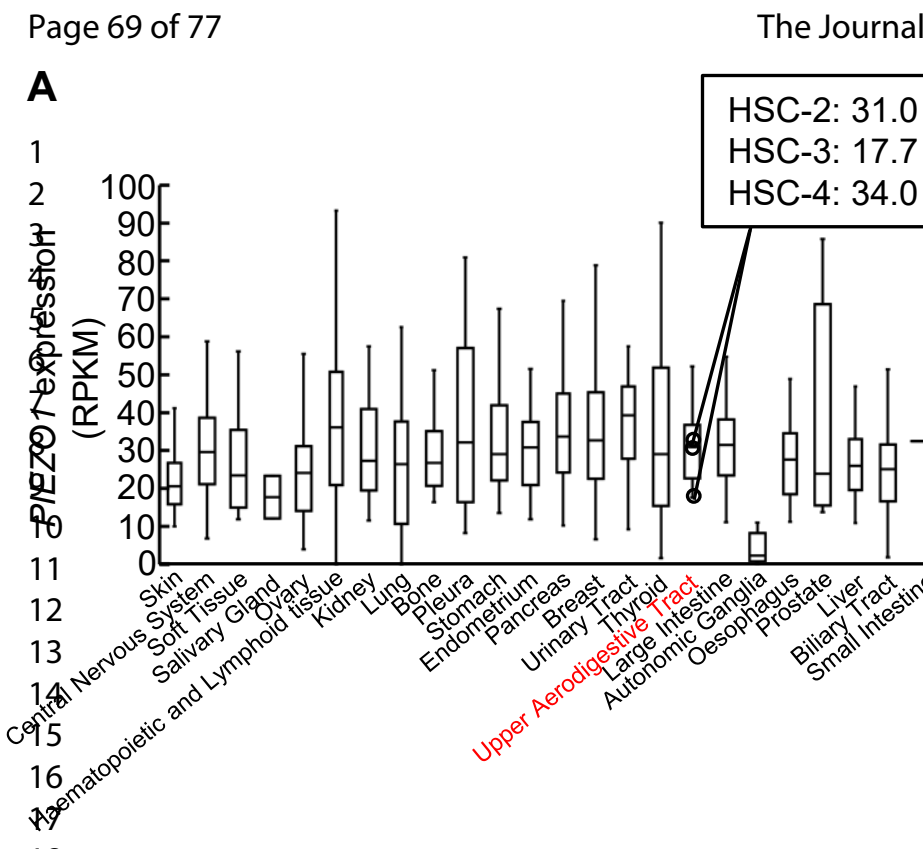

18

B

2
2
2
2
2
2
2
2
2
2
2
2
2

e

34

35

39

40

$41100=4$

4

43

44

4

46
47

48

49

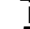

腥

5

5

5

54

55

56
D
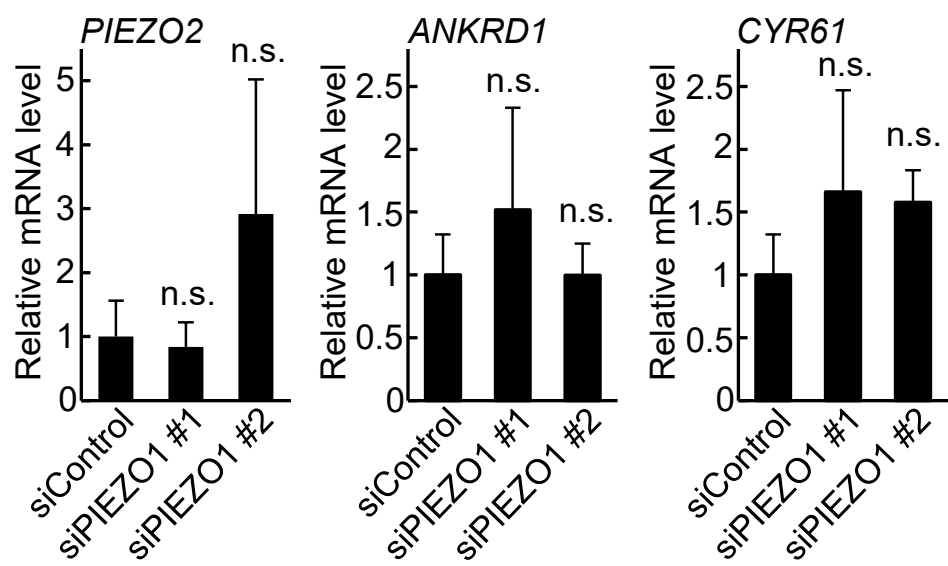

YAP/TAZ

Phalloidin
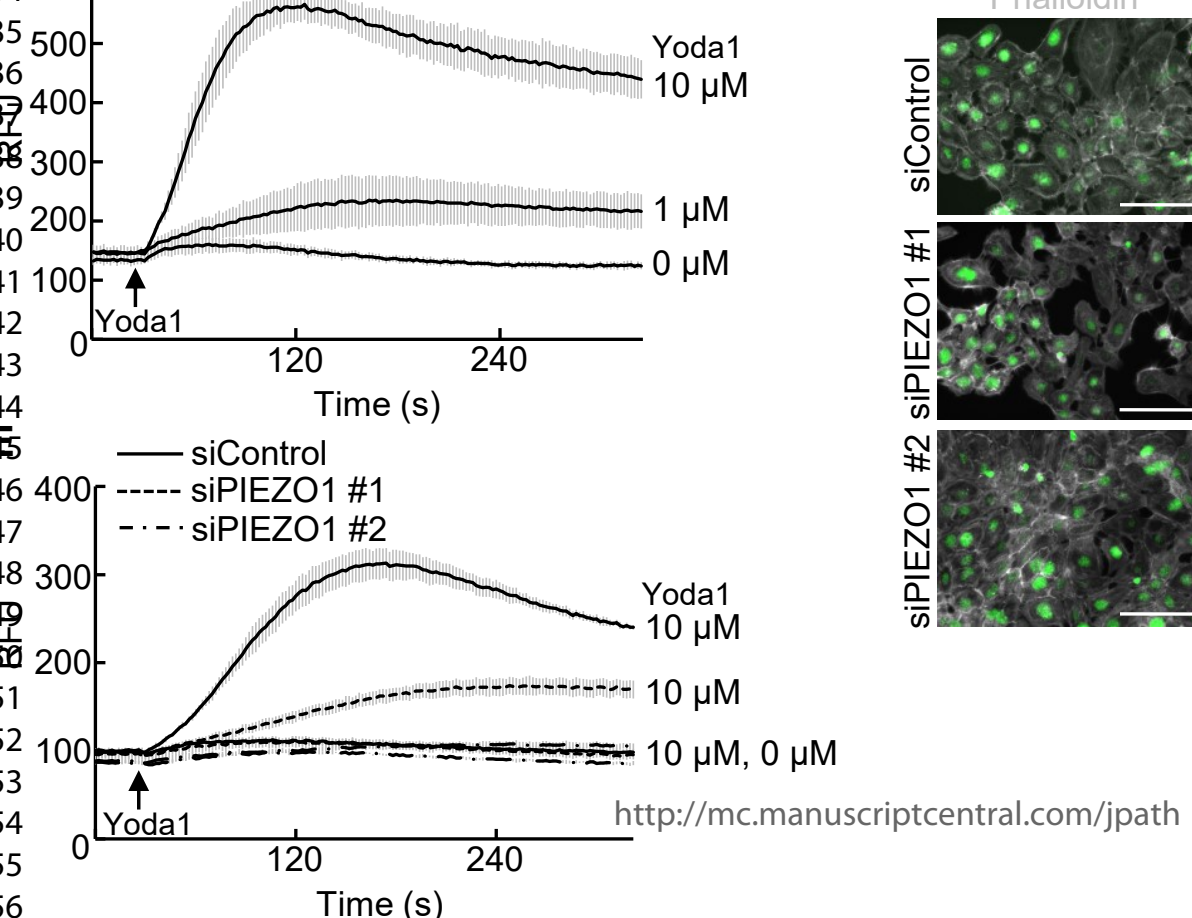

Yoda1

$10 \mu \mathrm{M}$

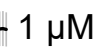

$0 \mu \mathrm{M}$

0 Yoda1

120

240



Time (s)

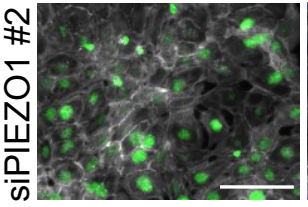

Hoechst 33342
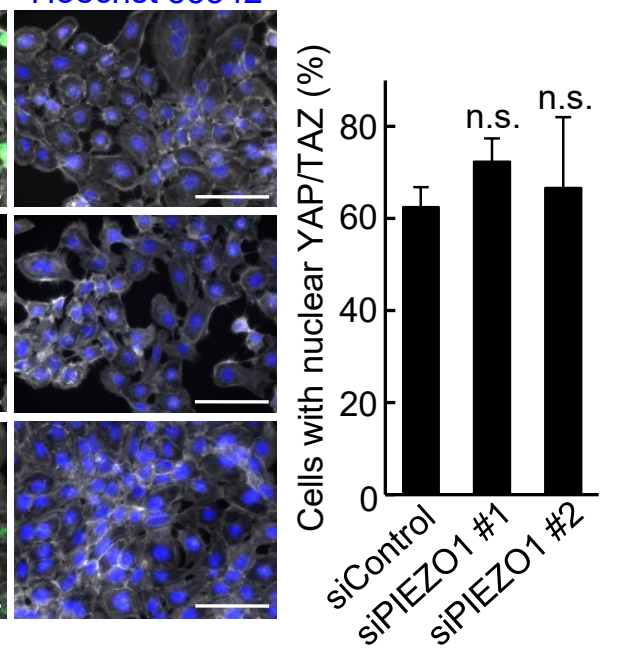

HSC-2: 0.009

HSC-3: 0.007

HSC-4: 0.047
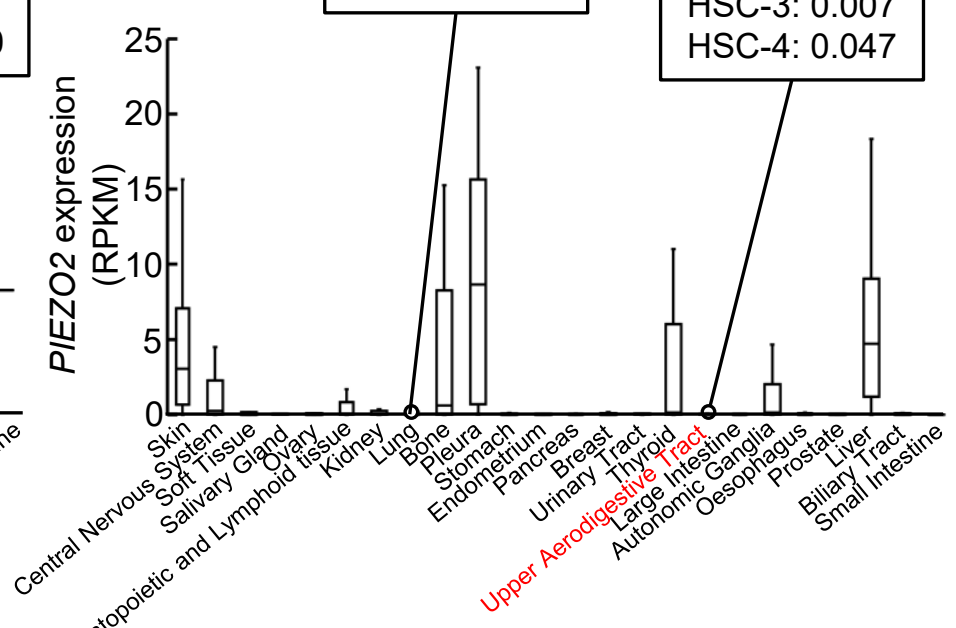
$1 \quad$ Supplementary Figure 3. The effect of PIEZO1 on YAP/TAZ signaling.

(A) The expression of PIEZO1 and PIEZO2 in cell lines was evaluated using Cancer Cell Line Encyclopedia dataset. (B) PIEZO2 mRNA levels were measured in OSCC cell lines (HSC-4, HSC-3, HSC-2, SAS, MISK81-5 and sMISK cells), A549 and MKN-45 cells by quantitative RTPCR. Relative levels of PIEZO2 mRNA expression were normalized to GAPDH and expressed as fold-changes compared with expression in control A549 cells. (C) HSC-4 cells were preloaded with Fluo-4-AM and were exposed without or with 1 and $10 \mu \mathrm{M}$ Yoda1. Then, intracellular $\mathrm{Ca}^{2+}$ influx was measured. RFU: relative fluorescence units. (D) HSC-4 cells were transfected with control or two different PIEZO1 siRNAs for $48 \mathrm{~h}$, and PIEZO2, ANKRD1 and CYR61 mRNA levels were measured by quantitative RT-PCR. Relative PIEZO2, ANKRD1 and CYR61 mRNA levels were normalized by GAPDH and expressed as fold-changes compared with levels in control siRNA transfected cells (upper panels). HSC-4 cells were transfected with control or two different PIEZO1 siRNAs for $48 \mathrm{~h}$, and the cells were stained with anti-YAP/TAZ antibody, phalloidin and Hoechst 33342. Cells with nuclear YAP/TAZ are expressed as the percentage of YAP/TAZ-positively stained cells compared with total Hoechst 33342-stained cells $(n=1516)$ (lower panels). (E) HSC-4 cells transfected with control or two different PIEZO1 siRNAs were pre-loaded with Fluo-4-AM and were exposed without or with $10 \mu \mathrm{M}$ Yoda1. Then, intracellular $\mathrm{Ca}^{2+}$ influx was measured. RFU: relative fluorescence units. Results are shown as means \pm s.d. of three independent experiments. Scale bars, $100 \mu \mathrm{m}$. n.s.: not significant. 
$1 \quad$ Supplementary Figure 4. PIEZO1 regulates cell proliferation of OSCC cells.

(A) HSC-4 cells were transfected with control or PIEZO1 \#1 siRNA for $48 \mathrm{~h}$. The cells were stained with anti-PCNA antibody and Hoechst 33342, and then PCNA-positive cells and Hoechst 33342-stained cells were counted, respectively. Results are expressed as the percentage of PCNA-positive cells compared with total Hoechst 33342-stained cells. (B) HSC-4 cells were transfected with control or PIEZO1 \#1 siRNA for $48 \mathrm{~h}$. The cells were stained with anti-cleaved caspase-3 antibody and DAPI. (C) HSC-4 cells expressing control or PIEZO1 \#1 and \#2 shRNAs were pre-loaded with Fluo-4-AM and were exposed without or with $10 \mu \mathrm{M}$ Yoda1. Then, intracellular $\mathrm{Ca}^{2+}$ influx was measured. RFU: relative fluorescence units. (D) HSC-4 cells expressing control or PIEZO1 \#1 and \#2 shRNAs were transfected with control or LATS2 siRNA for $48 \mathrm{~h}$ (left panels) or with mock or YAP ${ }^{5 \mathrm{SA}}$ expression (right panels), and then cells were cultured for 4 days in the 3D Matrigel. The cells were stained with anti-PCNA antibody and Hoechst 33342, and then PCNA-positive cells and Hoechst 33342-stained cells were counted, respectively. Results are expressed as the percentage of PCNA-positively stained cells compared with total Hoechst 33342-stained cells $(n=14,242)$. (E) HSC-4 cells expressing PIEZO1 \#1 and \#2 shRNAs were transfected with control or LATS2 siRNA for $48 \mathrm{~h}$ (left panels) or with mock or YAP ${ }^{5 \mathrm{SA}}$ expression (right panels), and then cells were cultured in $2 \mathrm{D}$ or suspension culture for $24 \mathrm{~h}$. PIEZO1 or ANKRD1 mRNA levels were measured by quantitative RT-PCR. Relative PIEZO1 or ANKRD1 mRNA levels were normalized by GAPDH and expressed as fold-changes compared with levels in 2D control cells. Results are shown as means \pm s.d. of three independent experiments. ${ }^{*} P<0.05,{ }^{*} P<0.01$. Scale bars, $100 \mu \mathrm{m}$. n.s.: not significant. 


\section{Hasegawa et al., Figure S5}

OSCC: Tumor lesion (Intensity of PIEZO1-positive signal)

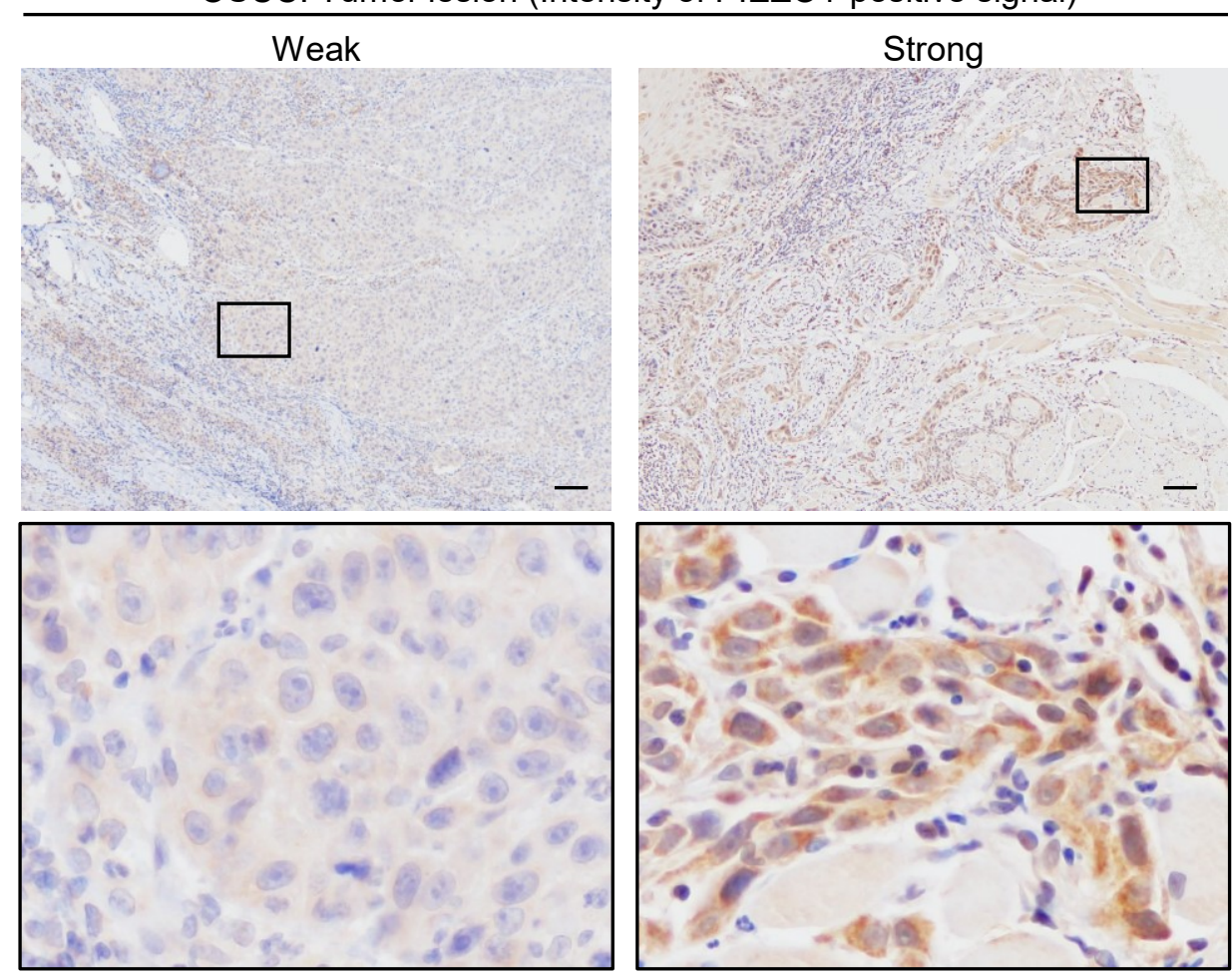

Non-tumor region (Intensity of PIEZO1-positive signal)

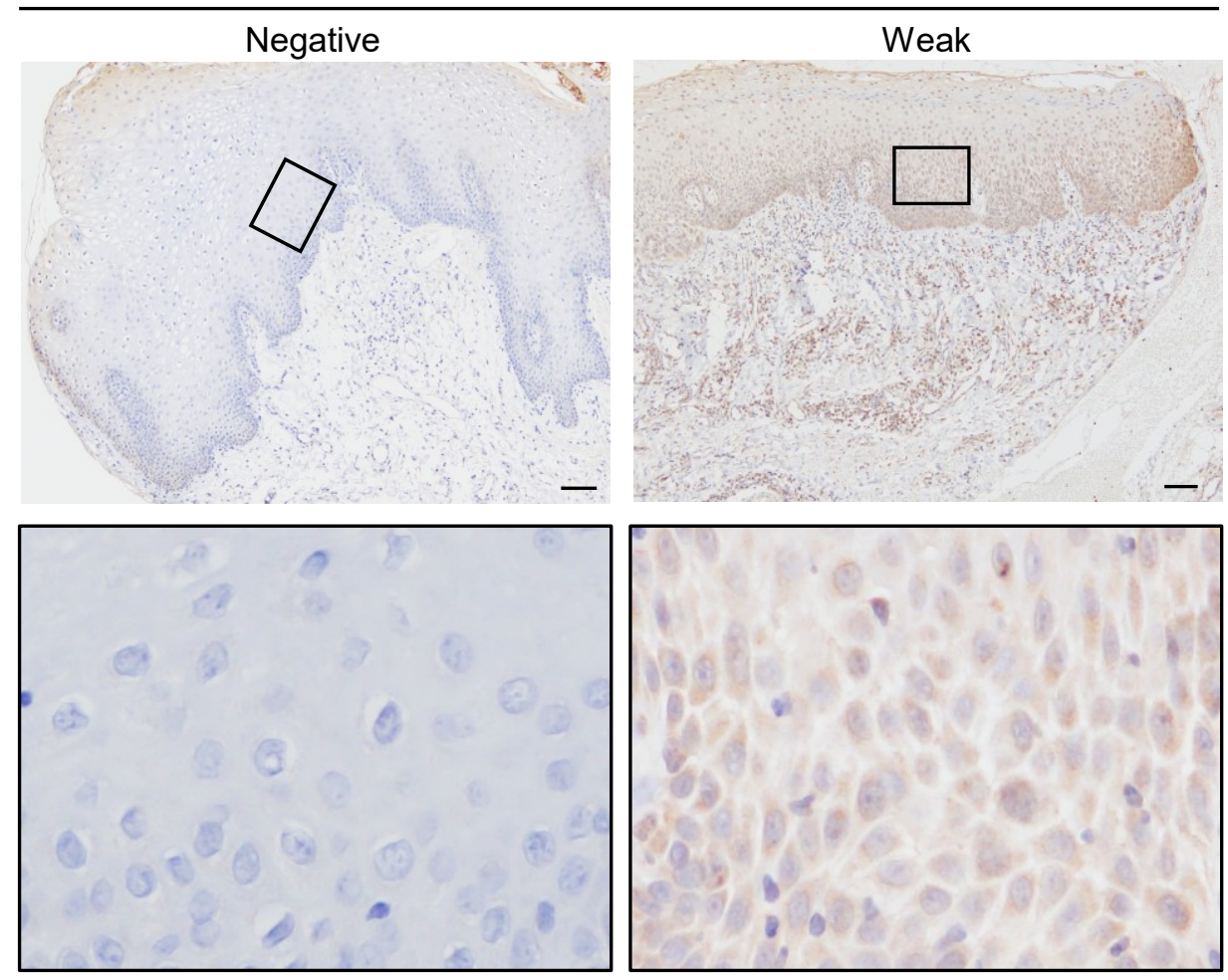

Supplementary Figure 5. PIEZO1 is expressed in human oral squamous cell carcinoma tissues.

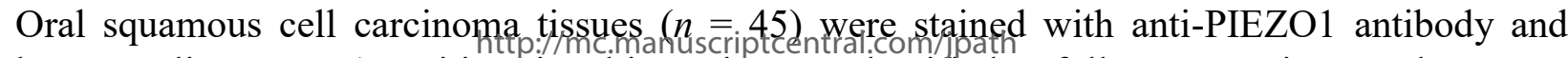
hematoxylin. PIEZO1-positive signal intensity was classified as follows: negative; weak; strong. Boxes show enlarged images. Scale bars, $100 \mu \mathrm{m}$. 
Hasegawa et al., Figure S6

1

7
5
6
7
8
9
1
1
1
1
1
1

$4 \quad$ Negative

8
0
1
2
3
4
5
6

\begin{tabular}{|l}
\hline$t$ \\
17 \\
18 \\
$1 s$ \\
2 \\
2 \\
2 \\
2 \\
2 \\
2 \\
26 \\
27 \\
28 \\
$\mathrm{~B}$
\end{tabular}

30

31

32

33

34

35

36

37

38

3

4

$4 \frac{1}{2}$.

4议

4 这

44

45

46

47

48

49

50

51

52

53

54

55

56

$\square$ Negative
OSCC: Tumor lesion (Expression and localization of YAP-positive signal)
Nucleus/Cytoplasm

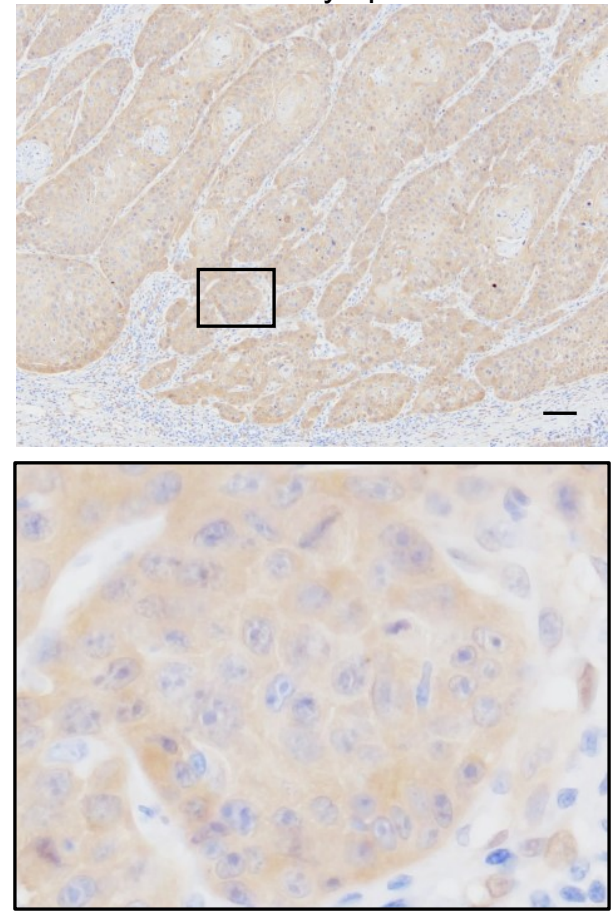

C

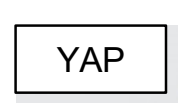

$\square$ Nucleus/Cytoplasm
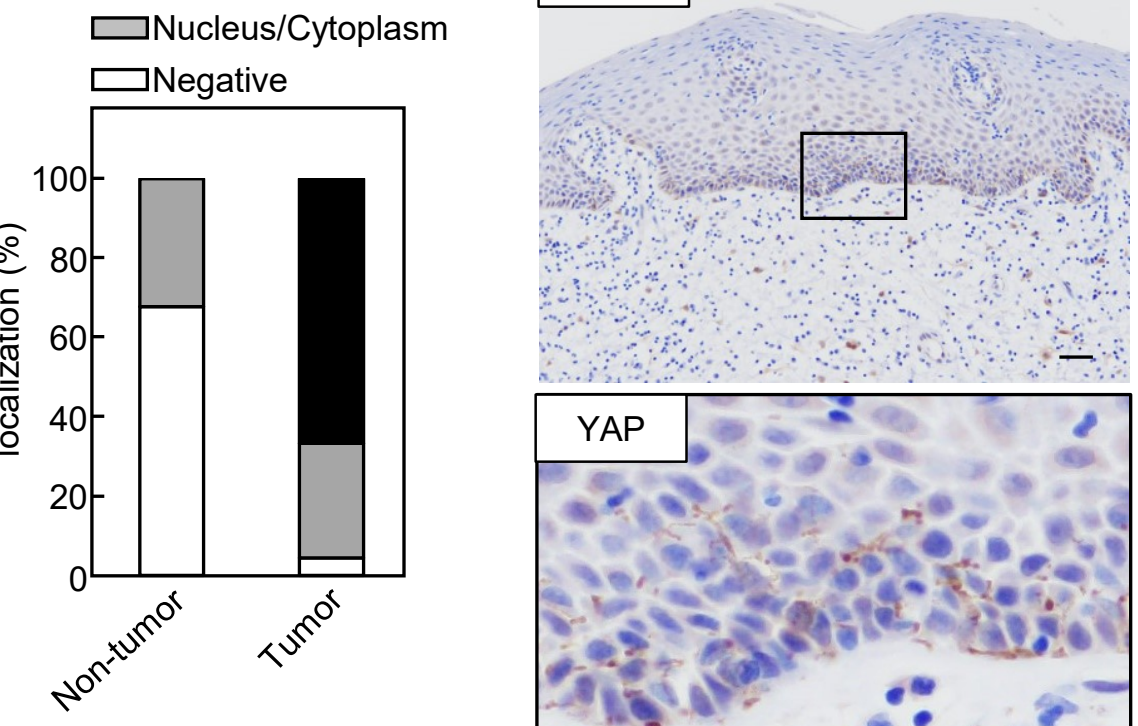

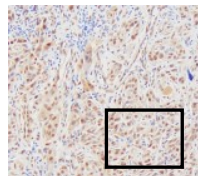

Nucleus
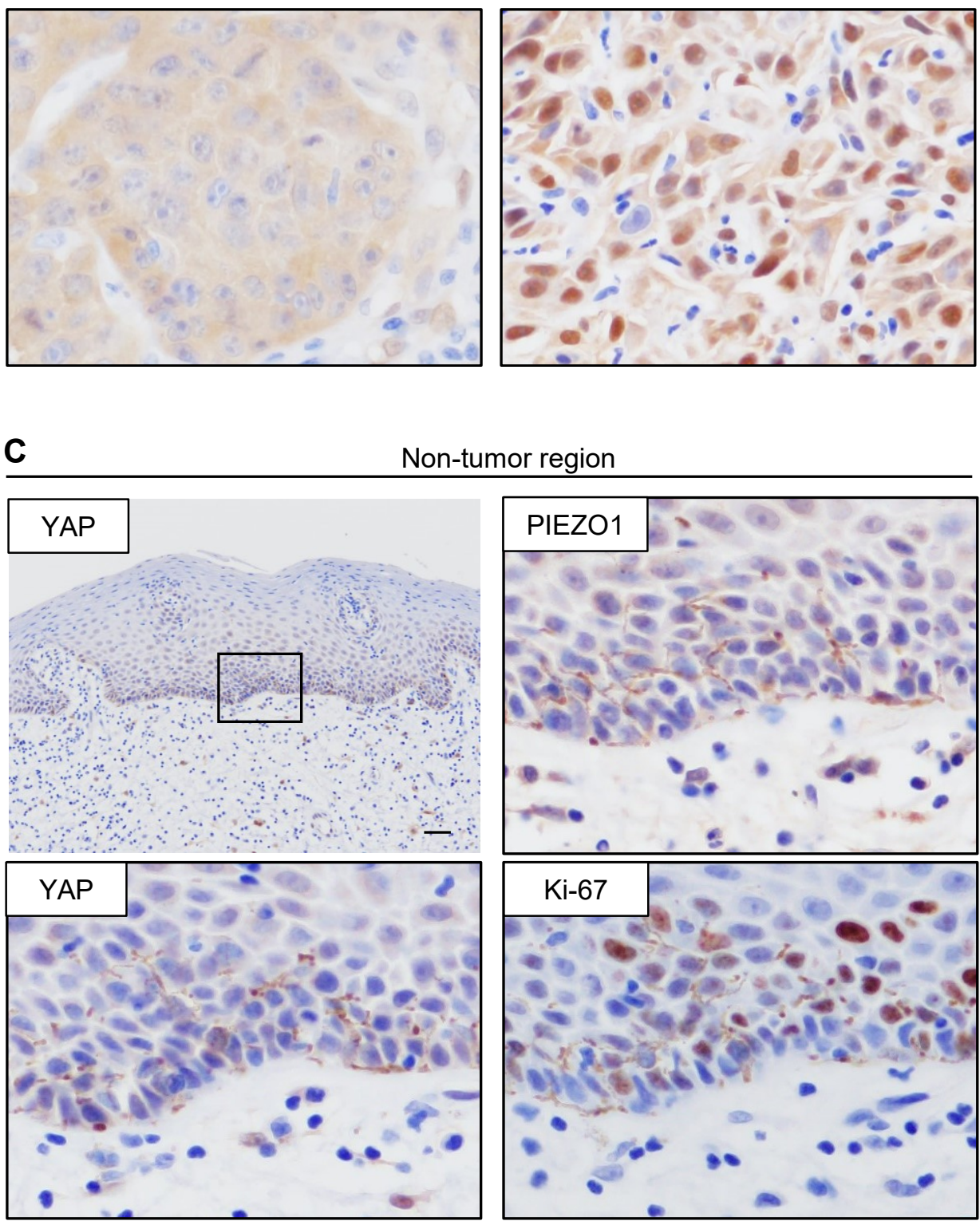

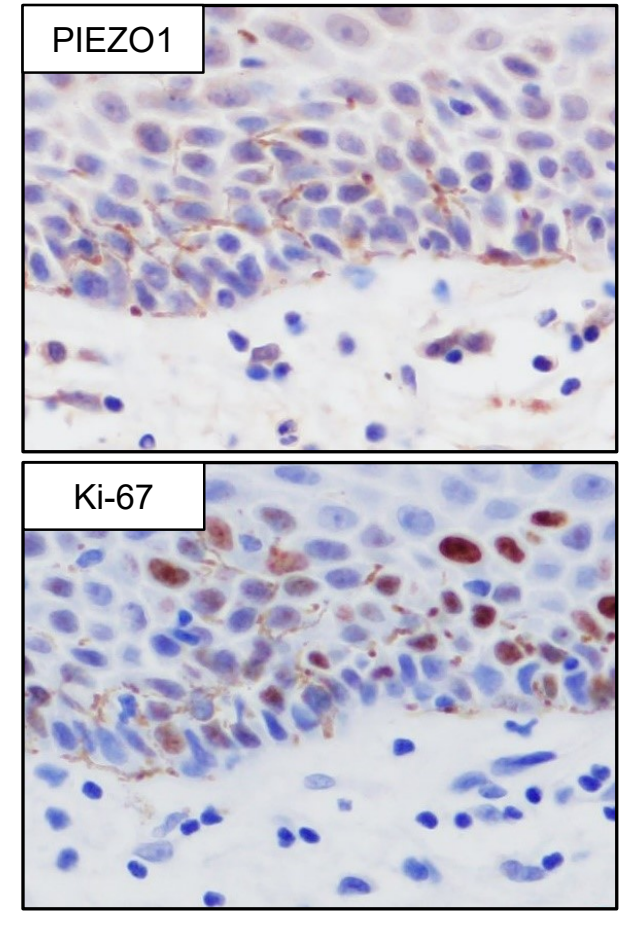

http://mc.manuscriptcentral.com/jpath



Non-tumor region 
$1 \quad$ Supplementary Figure 6. YAP is expressed in human oral squamous cell carcinoma tissues. (A) Oral squamous cell carcinoma tissues $(n=45)$ were stained with anti-YAP antibody and 3 hematoxylin. YAP expression and localization were classified as follows: negative;

4 nucleus/cytoplasm; nucleus. Boxes show enlarged images. (B) Percentages of three groups based 5 on the YAP expression and localization in the tumor lesions and the non-tumor regions are 6 shown in the panel. (C) Oral squamous cell carcinoma tissues $(n=45)$ were stained with anti8 YAP, anti-PIEZO1 and anti-Ki-67 antibodies, and hematoxylin. Box shows enlarged images. $9 \quad$ Scale bars, $50 \mu \mathrm{m}$. 
1

2

3

4

5

严

8

9

.

12

13

14

15

\#2

17

18

19

20

21

22

23

24

\#3

26

27

28

29

30

31

32

33

教

36

37

38

39

40

41

42

43

\#

45

46

47

48

49

50

51

52

53

54

55

OSCC: Tumor lesion
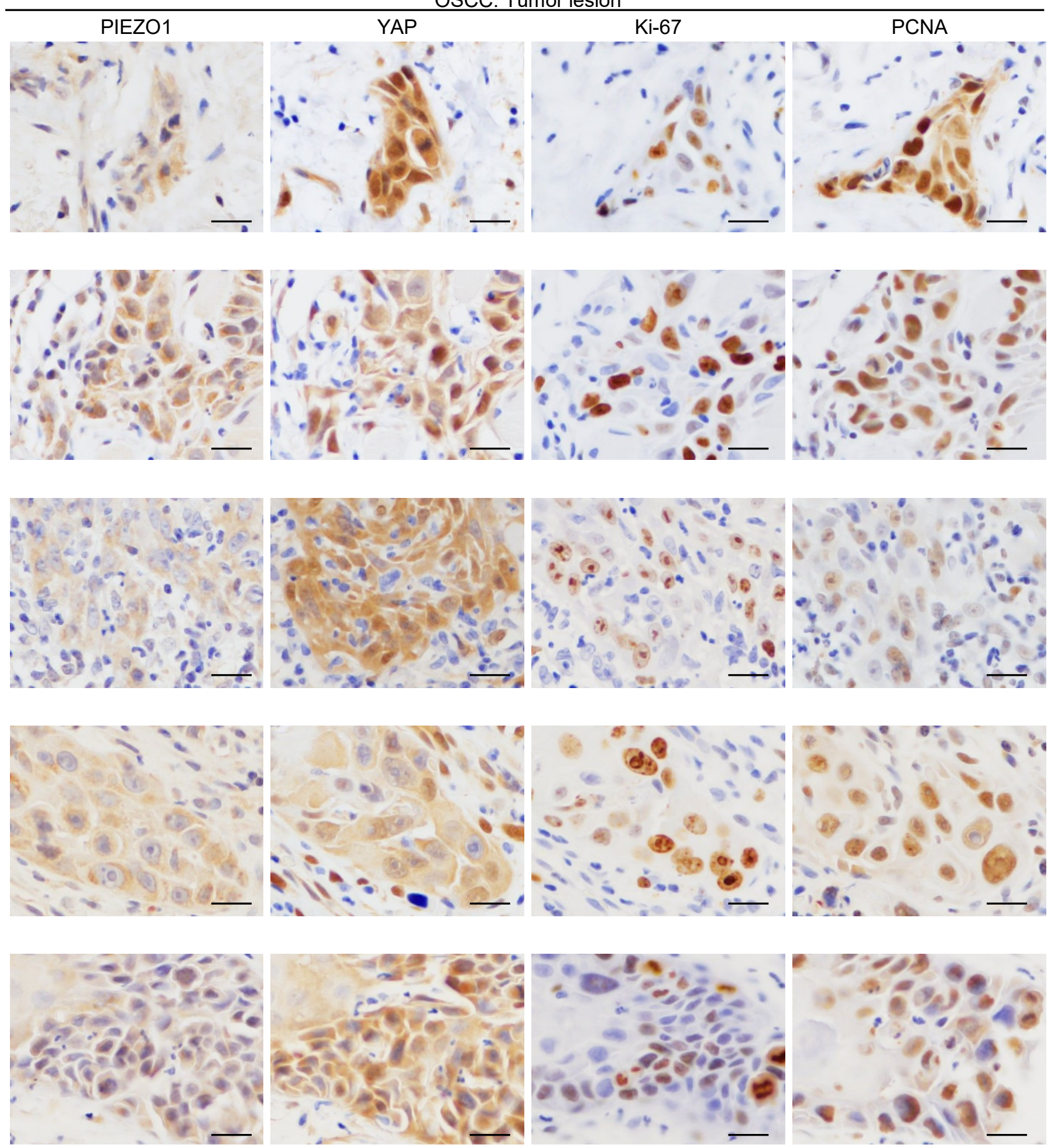

Supplementary Figure 7. Co-expression of PIEZO1, YAP, Ki-67 and PCNA in human oral squamous cell carcinoma tissues.

Oral squamous cell carcinoma tissues $(n=45)$ were stained with anti-PIEZO1, anti- YAP, anti$\mathrm{Ki}-67$ and anti-PCNA antibodies, and hematoxylin. Representative data $(\mathrm{n}=5)$ are shown. Scale bars, $20 \mu \mathrm{m}$.

http://mc.manuscriptcentral.com/jpath 

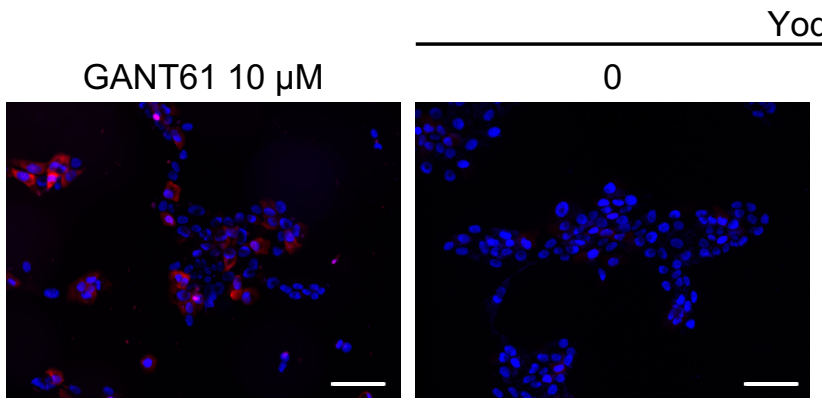

Yoda1

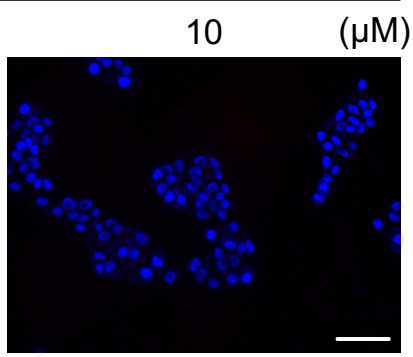

$\mathrm{Pl} /$ Hoechst 33342

Response to Reviewer \#1 Figure 1. The vitality of the cells treated with Yoda1. HSC-4 cells were treated with $10 \mu \mathrm{M}$ GANT61 in the absence of FBS for $24 \mathrm{~h}$, or without or with $10 \mu \mathrm{M}$ Yodal in the presence of $10 \%$ FBS for 5 min (see Figure 3B). The cells were stained with Propidium Iodide (PI) and Hoechst 33342. GANT61, a GLI-1 and GLI-2 inhibitor, was treated as a positive control [1]. Scale bars, $100 \mu \mathrm{m}$.

[1] Mikami Y, Fujii S, Nagata K, et al. GLI-mediated Keratin 17 expression promotes tumor cell growth through the anti-apoptotic function in oral squamous cell carcinomas. J Cancer Res Clin Oncol 2017; 143: 1381-1393. 